\title{
Generalized MICZ-Kepler Problems and Unitary Highest Weight Modules
}

\author{
Guowu Meng $1, *$ and Ruibin Zhang ${ }^{2,+}$ \\ ${ }^{1}$ Department of Mathematics, Hong Kong Univ. of Sci. and Tech., Clear Water Bay, Kowloon, Hong Kong \\ ${ }^{2}$ School of Mathematics and Statistics, University of Sydney Sydney, NSW 2006, Australia \\ For each integer $n \geq 1$, we demonstrate that a (2n+1)-dimensional generalized MICZ-Kepler prob- \\ lem has an $\operatorname{Spin}(2,2 n+2)$ dynamical symmetry which extends the manifest $\operatorname{Spin}(2 n+1)$ symmetry. \\ The Hilbert space of bound states is shown to form a unitary highest weight $\operatorname{Spin}(2,2 n+2)$-module \\ which occurs at the first reduction point in the Enright-Howe-Wallach classification diagram for the \\ unitary highest weight modules. As a byproduct, we get a simple geometric realization for such a \\ unitary highest weight $\operatorname{Spin}(2,2 n+2)$-module.
}

PACS numbers: 03.65.-w

\section{Contents}

\section{Introduction}

\section{Review of generalized MICZ-Kepler problems}

A. Generalized MICZ-Kepler problems

B. Basic identities for the gauge potential

III. The dynamical symmetry

IV. Representation theoretical aspects - the preliminary part

V. Representation theoretical aspects - the final part

A. $\tilde{\mathcal{H}}$ is a unitary highest weight Harish-Chandra module

B. Proof of Theorem 1

*Electronic address: mameng@ust.hk

${ }^{\dagger}$ Electronic address: rzhang@mail.usyd.edu.au 


\section{A. Geometrically transparent description}

References

\section{INTRODUCTION}

The Kepler problem is a physics problem in dimension three about two bodies which attract each other by a force proportional to the inverse square of their distance. As is well known, its exact solution in classical mechanics gives a very satisfactory explanation of the Kepler's laws of planetary motion, and its exact solution in quantum mechanics gives an equally satisfactory explanation of the spectral lines for the hydrogen atom. The MICZ-Kepler problems, discovered in the late $60 \mathrm{~s}$ by McIntosh and Cisneros [1] and independently by Zwanziger [2], are natural cousins of the Kepler problem. Roughly speaking, a MICZ-Kepler problem is the Kepler problem for which the nucleus of a hypothetic hydrogen atom also carries a magnetic charge.

In the early 90s, Iwai [3] obtained non-abelian analogues of the MICZ-Kepler problems in dimension five; more recently, the first author constructed and solved [4] analogues of the MICZ-Kepler problems in all dimensions bigger than or equal to three which extends the aforementioned work of McIntosh and Cisneros, Zwanziger, and Iwai. We shall refer to the MICZ-Kepler problems and their higher dimensional analogues as the generalized MICZ-Kepler problems.

Recall that the MICZ-Kepler problems all have a large dynamical symmetry group-Spin(2,4)-as shown by Barut and Bornzin [5]. These authors also used the symmetry to provide an elegant solution for the problems in [5]. Similar results were also established in dimension five by Pletyukhov and Tolkachev [6] for the generalized MICZKepler problems of Iwai. The purpose of the present paper is to investigate the dynamical symmetry and explore its representation theory for the generalized MICZ-Kepler problems in all odd dimensions.

We shall show that for each positive integer $n$, a $(2 n+1)$-dimensional generalized MICZ-Kepler problem always has an $\operatorname{Spin}(2,2 n+2)^{1}$ dynamical symmetry, i.e., its Hilbert space of bound states forms an irreducible unitary module for $\operatorname{Spin}(2,2 n+2)$. In fact, we shall show that the Hilbert space of bound states forms a unitary highest weight module for $\operatorname{Spin}(2,2 n+2)$; more precisely, we shall establish the following result ${ }^{2}$ :

Theorem 1. Assume $n \geq 1$ is an integer and $\mu$ is an half integer. Let $\mathscr{H}(\mu)$ be the Hilbert space of bound states for the $(2 n+1)$-dimensional generalized MICZ-Kepler problem with magnetic charge $\mu$, and $l_{\mu}=l+|\mu|+n-1$ for any integer $l \geq 0$.

1) There is a natural unitary action of $\operatorname{Spin}(2,2 n+2)$ on $\mathscr{H}(\mu)$ which extends the manifest unitary action of $\operatorname{Spin}(2 n+1)$. In fact, $\mathscr{H}(\mu)$ is the unitary highest weight module of $\operatorname{Spin}(2,2 n+2)$ with highest weight

\footnotetext{
${ }^{1}$ It is a double cover of $\mathrm{SO}_{0}(2,2 n+2)$ - the identity component of $\mathrm{SO}(2,2 n+2)$. By definition, it is characterized by the homomorphism $\pi_{1}\left(\mathrm{SO}_{0}(2,2 n+2)\right)=\mathbb{Z} \oplus \mathbb{Z}_{2} \rightarrow \mathbb{Z}_{2}$ which maps $(a, b)$ to $\bar{a}+b$. Here $\bar{a}$ is the congruence class of $a$ modulo 2.

2 The result for an even dimensional generalized MICZ-Kepler problem shall be presented in Ref. [7]
} 
$(-(n+|\mu|),|\mu|, \cdots,|\mu|, \mu)$; consequently, it occurs at the first reduction point of the Enright-Howe-Wallach classification diagram ${ }^{3}$ for the unitary highest weight modules, so it is a non-discrete series representation.

2) As a representation of subgroup $\operatorname{Spin}(2,1) \times_{\mathbb{Z}_{2}} \operatorname{Spin}(2 n+1)$,

$$
\mathscr{H}(\mu)=\hat{\bigoplus}_{l=0}^{\infty}\left(\mathcal{D}_{2 l_{\mu}+2}^{-} \otimes D_{l}\right)
$$

where $D_{l}$ is the irreducible module of $\operatorname{Spin}(2 n+1)$ with highest weight $(l+|\mu|,|\mu|, \cdots,|\mu|)$ and $\mathcal{D}_{2 l_{\mu}+2}^{-}$is the antiholomorphic discrete series representation of $\operatorname{Spin}(2,1)$ with highest weight $-l_{\mu}-1$.

3) As a representation of the maximal compact subgroup $\operatorname{Spin}(2) \times_{\mathbb{Z}_{2}} \operatorname{Spin}(2 n+2)$,

$$
\mathscr{H}(\mu)=\hat{\bigoplus}_{l=0}^{\infty}\left(D\left(-l_{\mu}-1\right) \otimes D^{l}\right)
$$

where $D^{l}$ is the irreducible module of $\operatorname{Spin}(2 n+2)$ with highest weight $(l+|\mu|,|\mu|, \cdots,|\mu|, \mu)$ and $D\left(-l_{\mu}-1\right)$ is the irreducible module of $\operatorname{Spin}(2)$ with weight $-l_{\mu}-1$.

Readers who wish to have a quick geometric description of the aforementioned unitary highest weight module of $\operatorname{Spin}(2,2 n+2)$ may consult the appendix. Readers who wish to know more details about the classification [8, 9, 10] of unitary highest weight modules may start with a fairly readable account from Ref. [10]. Note that there is no general classification result for the family of unitary modules of real non-compact simple Lie groups, and the subfamily of unitary highest weight modules is special enough so that such a nice classification result can possibly exist. The first reduction point picked up by the "Nature" from the Enright-Howe-Wallach classification diagram is even more special because it belongs to an even more special subfamily called Wallach set.

In section [I] we give a quick review of the generalized MICZ-Kepler problems in odd dimensions. For the computational purpose in the subsequent section, we quickly review the gauge potential ${ }^{4}$ for the background gauge field (i.e., connection) under a particular local gauge (i.e., bundle trivialization), and then quote from Ref. [4] some key identities satisfied by the gauge potential. In section III, we introduce the dynamical symmetry operators and show that they satisfy the commutation relations for the generators ${ }^{5}$ of $\mathfrak{s o}_{0}(2,2 n+2)$. We also show that these dynamical symmetry operators satisfy a set of quadratic relations ${ }^{6}$. In section IV we start with a preliminary discussion of the representation problem and point out the need of "twisting". Then we gave a review of the (bound) energy eigenspaces (i.e., eigenspaces of the harmiltonian viewed as a hermitian operator on the physical Hilbert space) and finally introduce the notion of "twisted" energy eigenspaces which is soon shown to be the space of $L^{2}$-sections of a canonical hermitian bundle. In the last section, we solve the representation problem by proving two propositions from which Theorem 1 follows quickly. In the appendix, each of the unitary highest weight representation of $\operatorname{Spin}(2,2 n+2)$

\footnotetext{
${ }^{3}$ Page 101, Ref. [10]. See also Refs. [8, 9].

${ }^{4}$ It is $\sqrt{-1}$ times the local connection one-form.

${ }^{5}$ Here we adopt the practice in physics: the Lie algebra generators act as hermitian operators in all unitary representations.

6 This set of quadratic relations will be shown [11] to algebraically characterize the unitary highest weight $\operatorname{Spin}(2,2 n+2)$-modules stated in Theorem 1 above.
} 
encountered here is geometrically realized as the space of all $L^{2}$ sections of a canonical hermitian bundle. Via communications with Profs. R. Howe and N. Wallach, we learned that these representations can be imbedded into the kernel of certain canonical differential operators, see Refs. [12, 13] for the case $\mu=0$ and Ref. [14] for the general case. Prof. Feher informed us of Ref. [15] in which a related interesting model with a conjectured dynamical $O(2,4)$ symmetry is investigated.

\section{REVIEW OF GENERALIZED MICZ-KEPLER PROBLEMS}

From the physics point of view, a MICZ-Kepler problem is a generalization of the Kepler problem by adding a suitable background magnetic field, while at the same time making an appropriate adjustment to the scalar Coulomb potential so that the problem is still integrable. The configuration space is the punctured 3D Euclidean space, and the background magnetic field is a Dirac monopole. To be more precise, the (dimensionless) hamiltonian of a MICZ-Kepler problem with magnetic charge $\mu$ is

$$
H=-\frac{1}{2} \Delta_{\mathcal{A}}+\frac{\mu^{2}}{2 r^{2}}-\frac{1}{r} .
$$

Here $\Delta_{\mathcal{A}}$ is the Laplace operator twisted by the gauge potential $\mathcal{A}$ of a Dirac monopole under a particular gauge, and $\mu$ is the magnetic charge of the Dirac monopole, which must be a half integer.

To extend the MICZ-Kepler problems beyond dimension three, one needs a suitable generalization of the Dirac monopoles. Fortunately this problem was solved in Refs. [4, 16, 17]. We review the work here.

\section{A. Generalized MICZ-Kepler problems}

Let $D \geq 3$ be an integer, $\mathbb{R}_{*}^{D}$ be the punctured $D$-space, i.e., $\mathbb{R}^{D}$ with the origin removed. Let $d s^{2}$ be the cylindrical metric on $\mathbb{R}_{*}^{D}$. Then $\left(\mathbb{R}_{*}^{D}, d s^{2}\right)$ is the product of the straight line $\mathbb{R}$ with the round sphere $\mathrm{S}^{D-1}$. Since we are interested in the odd dimensional generalized MICZ-Kepler problems only in this paper, we assume $D$ is odd.

Let $\mathcal{S}_{ \pm}$be the positive/negative spinor bundle of $\left(\mathbb{R}_{*}^{D}, d s^{2}\right)$, then $\mathcal{S}_{ \pm}$correspond to the fundamental spin representations $\mathbf{s}_{ \pm}$of $\mathfrak{s o}_{0}(D-1)$ (the Lie algebra of $\operatorname{SO}(D-1)$ ). Note that each of the above spinor bundles is endowed with a natural $\mathrm{SO}(D)$ invariant connection — the Levi-Civita spin connection of $\left(\mathbb{R}_{*}^{D}, d s^{2}\right)$. As a result, the Young product of $I$ copies of these bundles, denoted by $\mathcal{S}_{+}^{I}, \mathcal{S}_{-}^{I}$ respectively, are also equipped with natural $\mathrm{SO}(D)$ invariant connections.

When $\mu$ is a positive half integer, we write $\mathcal{S}_{+}^{2 \mu}$ as $\mathcal{S}^{2 \mu}$, and $\mathcal{S}_{-}^{2 \mu}$ as $\mathcal{S}^{-2 \mu}$. We also adopt this convention for $\mu=0$ : to denote by $\mathcal{S}^{0}$ the product complex line bundle with the product connection. Note that $\mathcal{S}^{2 \mu}$ with $\mu$ being a half integer is our analogue of the Dirac monopole with magnetic charge $\mu$, and the corresponding representation of $\mathfrak{s o}_{0}(D-1)$ will be denoted by $\mathbf{s}^{2 \mu}$. 
Definition 1. Let $n \geq 1$ be an integer, $\mu$ a half integer. The $(2 n+1)$-dimensional generalized MICZ-Kepler problem with magnetic charge $\mu$ is defined to be the quantum mechanical system on $\mathbb{R}_{*}^{2 n+1}$ for which the wave-functions are sections of $\mathcal{S}^{2 \mu}$, and the hamiltonian is

$$
H=-\frac{1}{2} \Delta_{\mu}+\frac{(n-1)|\mu|+\mu^{2}}{2 r^{2}}-\frac{1}{r}
$$

where $\Delta_{\mu}$ is the Laplace operator twisted by $\mathcal{S}^{2 \mu}$.

Upon choosing a local gauge, the background gauge field (i.e., the natural connection on $\mathcal{S}^{2 \mu}$ ) can be represented by a gauge potential $\mathcal{A}_{\alpha}$ in an explicit form; then $\Delta_{\mu}$ can be represented explicitly by $\sum_{\alpha}\left(\partial_{\alpha}+i \mathcal{A}_{\alpha}\right)^{2}$. Since the gauge potential is of crucial importance, we review some of its properties in the next subsection.

\section{B. Basic identities for the gauge potential}

We write $\vec{r}=\left(x_{1}, x_{2}, \ldots, x_{D-1}, x_{D}\right)$ for a point in $\mathbb{R}^{D}$ and $r$ for the length of $\vec{r}$. The small Greek letters $\mu, \nu$, etc run from 1 to $D$ and the lower case Latin letters $a, b$ etc run from 1 to $D-1$. We use the Einstein convention that repeated indices are always summed over.

Under a suitable choice of local gauge on $\mathbb{R}^{D}$ with the negative $D$-th axis removed, the gauge field can be represented by the following gauge potential:

$$
\mathcal{A}_{D}=0, \quad \mathcal{A}_{b}=-\frac{1}{r\left(r+x_{D}\right)} x_{a} \gamma_{a b}
$$

where $\gamma_{a b}=\frac{i}{4}\left[\gamma_{a}, \gamma_{b}\right]$ with $\gamma_{a}$ being the "gamma matrix" for physicists. Note that $\gamma_{a}=i e_{a}$ with $e_{a}$ being the element in the Clifford algebra that corresponds to the $a$-th standard coordinate vector of $\mathbb{R}^{D-1}$.

The field strength of $\mathcal{A}_{\alpha}$ is then given by

$$
\begin{aligned}
F_{D b}= & \frac{1}{r^{3}} x_{a} \gamma_{a b}, \\
F_{a b}= & -\frac{2 \gamma_{a b}}{r\left(r+x_{D}\right)}+\frac{1}{r^{2}\left(r+x_{D}\right)^{2}} . \\
& \left(\left(2+\frac{x_{D}}{r}\right) x_{c}\left(x_{a} \gamma_{c b}-x_{b} \gamma_{c a}\right)+i x_{d} x_{c}\left[\gamma_{d a}, \gamma_{c b}\right]\right)
\end{aligned}
$$

Here are some identities from Ref. [4] that our later computations will crucially depend on:

Lemma 1. Let $\mathcal{A}_{\alpha}$ be the gauge potential defined by equation(5) and let $F_{\alpha \beta}$ be its field strength.

1) The following identities are valid in any representation of $\mathfrak{s o}_{0}(D-1)$ :

$$
\begin{array}{r}
F_{\mu \nu} F^{\mu \nu}=\frac{2}{r^{4}} c_{2}, \quad\left[\nabla_{\kappa}, F_{\mu \nu}\right]=\frac{1}{r^{2}}\left(x_{\mu} F_{\nu \kappa}+x_{\nu} F_{\kappa \mu}-2 x_{\kappa} F_{\mu \nu}\right), \\
x_{\mu} \mathcal{A}_{\mu}=0, \quad x_{\mu} F_{\mu \nu}=0, \quad\left[\nabla_{\mu}, F_{\mu \nu}\right]=0, \\
r^{2}\left[F_{\mu \nu}, F_{\alpha \beta}\right]+i F_{\mu \beta} \delta_{\alpha \nu}-i F_{\nu \beta} \delta_{\alpha \mu}+i F_{\alpha \mu} \delta_{\beta \nu}-i F_{\alpha \nu} \delta_{\beta \mu} \\
=\frac{i}{r^{2}}\left(x_{\mu} x_{\alpha} F_{\beta \nu}+x_{\mu} x_{\beta} F_{\nu \alpha}-x_{\nu} x_{\alpha} F_{\beta \mu}-x_{\nu} x_{\beta} F_{\mu \alpha}\right),
\end{array}
$$


where $\nabla_{\alpha}=\partial_{\alpha}+i \mathcal{A}_{\alpha}$, and $c_{2}=c_{2}\left[\mathfrak{s o}_{0}(D-1)\right]=\frac{1}{2} \gamma_{a b} \gamma_{a b}$ is the (quadratic) Casimir operator of $\mathfrak{s o}_{0}(D-1)$.

2) When $D=2 n+1, \mu$ is a half integer, the following identity

$$
r^{2} F_{\lambda \alpha} F_{\lambda \beta}=\frac{c_{2}}{n}\left(\frac{1}{r^{2}} \delta_{\alpha \beta}-\frac{x_{\alpha} x_{\beta}}{r^{4}}\right)+i(n-1) F_{\alpha \beta}
$$

holds in the irreducible representation $\mathbf{s}^{2 \mu}$ of $\mathfrak{s o}_{0}(2 n)$ with highest weight $(|\mu|, \cdots,|\mu|, \mu)$.

Note that $\frac{c_{2}}{n}=\mu^{2}+(n-1)|\mu|$ in the irreducible representation $\mathbf{s}^{2 \mu}$. Remark that $\mathcal{A}_{r}=\mathcal{A}_{\theta}=0$, where $\mathcal{A}_{r}$ and $\mathcal{A}_{\theta}$ are the $r$ and $\theta$ components of $\mathcal{A}$ in the polar coordinate system $\left(r, \theta, \theta_{1}, \cdots, \theta_{D-3}, \phi\right)$ for $\mathbb{R}_{*}^{D}$ with $\theta$ being the angle between $\vec{r}$ and the positive $D$-th axis.

\section{THE DYNAMICAL SYMMETRY}

For the remainder of this paper, we only consider a fixed $(2 n+1)$-dimensional generalized MICZ-Kepler problem with magnetic charge $\mu$. Recall that the configuration space is $\mathbb{R}_{*}^{D}$ where $D=2 n+1$. For our computational purposes, it suffices to work on $\mathbb{R}^{D}$ with the negative $D$-axis removed. Introduce the notations $\pi_{\alpha}:=-i \nabla_{\alpha}, c:=\mu^{2}+(n-1)|\mu|$. Then $\left[\pi_{\alpha}, \pi_{\beta}\right]=-i F_{\alpha \beta}$.

Following Barut and Bornzin [5], we let

$$
\left\{\begin{array}{l}
\vec{\Gamma}:=r \vec{\pi}, \quad X:=r \pi^{2}+\frac{c}{r}, \quad Y:=r \\
J_{\alpha \beta}:=i\left[\Gamma_{\alpha}, \Gamma_{\beta}\right], \quad \vec{Z}:=i[\vec{\Gamma}, X], \quad \vec{W}:=i[\vec{\Gamma}, Y]=\vec{r}
\end{array}\right.
$$

and

$$
\left\{\begin{array}{l}
\Gamma_{D+1}:=\frac{1}{2}(X-Y), \quad \Gamma_{-1}:=\frac{1}{2}(X+Y), \\
\vec{A}:=\frac{1}{2}(\vec{Z}-\vec{W}), \quad \vec{M}:=\frac{1}{2}(\vec{Z}+\vec{W}), \quad T:=i\left[\Gamma_{D+1}, \Gamma_{-1}\right] .
\end{array}\right.
$$

Some relatively straightforward but lengthy computations yield

$$
\left\{\begin{aligned}
J_{\alpha \beta} & =x_{\alpha} \pi_{\beta}-x_{\beta} \pi_{\alpha}+r^{2} F_{\alpha \beta}, \\
A_{\alpha} & =\frac{1}{2} x_{\alpha} \pi^{2}-\pi_{\alpha}(\vec{r} \cdot \vec{\pi})+r^{2} F_{\alpha \beta} \pi_{\beta}-\frac{c}{2 r^{2}} x_{\alpha}+\frac{i}{2}(D-3) \pi_{\alpha}-\frac{1}{2} x_{\alpha}, \\
M_{\alpha} & =\frac{1}{2} x_{\alpha} \pi^{2}-\pi_{\alpha}(\vec{r} \cdot \vec{\pi})+r^{2} F_{\alpha \beta} \pi_{\beta}-\frac{c}{2 r^{2}} x_{\alpha}+\frac{i}{2}(D-3) \pi_{\alpha}+\frac{1}{2} x_{\alpha} \\
T & =\vec{r} \cdot \vec{\pi}-i \frac{D-1}{2} \\
\Gamma_{\alpha} & =r \pi_{\alpha} \\
\Gamma_{-1} & =\frac{1}{2}\left(r \pi^{2}+r+\frac{c}{r}\right) \\
\Gamma_{D+1} & =\frac{1}{2}\left(r \pi^{2}-r+\frac{c}{r}\right) .
\end{aligned}\right.
$$


Let the capital Latin letters $A, B$ run from -1 to $D+1$. Introduce $J_{A B}$ as follows:

$$
J_{A B}= \begin{cases}J_{\mu \nu} & \text { if } A=\mu, B=\nu \\ A_{\mu} & \text { if } A=\mu, B=D+1 \\ M_{\mu} & \text { if } A=\mu, B=-1 \\ \Gamma_{\mu} & \text { if } A=\mu, B=0 \\ T & \text { if } A=D+1, B=-1 \\ \Gamma_{D+1} & \text { if } A=D+1, B=0 \\ \Gamma_{-1} & \text { if } A=-1, B=0 \\ -J_{B A} & \text { if } A>B \\ 0 & \text { if } A=B .\end{cases}
$$

Theorem 2. Let $C^{\infty}\left(\mathcal{S}^{2 \mu}\right)$ be the space of smooth sections of $\mathcal{S}^{2 \mu}$. Let $J_{A B}$ be defined by (12).

1) As operators on $C^{\infty}\left(\mathcal{S}^{2 \mu}\right), J_{A B}$ 's satisfy the following commutation relations:

$$
\left[J_{A B}, J_{A^{\prime} B^{\prime}}\right]=-i \eta_{A A^{\prime}} J_{B B^{\prime}}-i \eta_{B B^{\prime}} J_{A A^{\prime}}+i \eta_{A B^{\prime}} J_{B A^{\prime}}+i \eta_{B A^{\prime}} J_{A B^{\prime}}
$$

where the indefinite metric tensor $\eta$ is $\operatorname{diag}\{++-\cdots-\}$ relative to the following order: $-1,0,1, \ldots, 2 n+2$ for the indices.

2) As operators on $C^{\infty}\left(\mathcal{S}^{2 \mu}\right)$,

$$
\left\{J_{A B}, J_{C}^{A}\right\}:=J_{A B} J_{C}^{A}+J^{A}{ }_{C} J_{A B}=-2 a \eta_{B C}
$$

where $a=n-c$.

The proof of this theorem is purely algebraic and computational, but quite long. It will be carried out in the next two subsections.

\section{A. Proof of part 1)}

By exploiting the symmetry properties of both sides of Eq. (13), we can see that it suffices to verify the commutation relations in the cases where $(A, B) \neq\left(A^{\prime}, B^{\prime}\right), A<B, A^{\prime}<B^{\prime}$ and $B^{\prime} \leq B$. The proof crucially depends on Lemma 1 .

The following lemma is quite useful.

\section{Lemma 2.}

$$
\left\{\begin{aligned}
{\left[J_{\alpha \beta}, r\right] } & =\left[J_{\alpha \beta}, \frac{1}{r}\right]=0 \\
{\left[J_{\alpha \beta}, x_{\nu}\right] } & =-i\left(x_{\alpha} \delta_{\beta \nu}-x_{\beta} \delta_{\alpha \nu}\right), \\
{\left[J_{\alpha \beta}, \pi_{\nu}\right] } & =-i\left(\pi_{\alpha} \delta_{\beta \nu}-\pi_{\beta} \delta_{\alpha \nu}\right), \\
{\left[J_{\alpha \beta}, F_{\alpha^{\prime} \beta^{\prime}}\right] } & =i \delta_{\alpha \alpha^{\prime}} F_{\beta \beta^{\prime}}+i \delta_{\beta \beta^{\prime}} F_{\alpha \alpha^{\prime}}-i \delta_{\alpha \beta^{\prime}} F_{\beta \alpha^{\prime}}-i \delta_{\beta \alpha^{\prime}} F_{\alpha \beta^{\prime}} .
\end{aligned}\right.
$$


Proof.

$$
\begin{aligned}
& {\left[J_{\alpha \beta}, r\right]=\left[x_{\alpha} \pi_{\beta}-x_{\beta} \pi_{\alpha}+r^{2} F_{\alpha \beta}, r\right]=\left[x_{\alpha} \pi_{\beta}-x_{\beta} \pi_{\alpha}, r\right]} \\
& =-i\left(x_{\alpha} \frac{x_{\beta}}{r}-x_{\beta} \frac{x_{\alpha}}{r}\right)=0 . \\
& {\left[J_{\alpha \beta}, \frac{1}{r}\right]=\left[x_{\alpha} \pi_{\beta}-x_{\beta} \pi_{\alpha}+r^{2} F_{\alpha \beta}, \frac{1}{r}\right]=\left[x_{\alpha} \pi_{\beta}-x_{\beta} \pi_{\alpha}, \frac{1}{r}\right]} \\
& =+i\left(x_{\alpha} \frac{x_{\beta}}{r^{3}}-x_{\beta} \frac{x_{\alpha}}{r^{3}}\right)=0 . \\
& {\left[J_{\alpha \beta}, x_{\nu}\right]=\left[x_{\alpha} \pi_{\beta}-x_{\beta} \pi_{\alpha}+r^{2} F_{\alpha \beta}, x_{\nu}\right]=\left[x_{\alpha} \pi_{\beta}-x_{\beta} \pi_{\alpha}, x_{\nu}\right]} \\
& =-i\left(x_{\alpha} \delta_{\beta \nu}-x_{\beta} \delta_{\alpha \nu}\right) . \\
& {\left[J_{\alpha \beta}, \pi_{\nu}\right]=\left[x_{\alpha} \pi_{\beta}-x_{\beta} \pi_{\alpha}+r^{2} F_{\alpha \beta}, \pi_{\nu}\right]} \\
& =-i\left(\pi_{\alpha} \delta_{\beta \nu}-\pi_{\beta} \delta_{\alpha \nu}\right)-i x_{\alpha} F_{\beta \nu}+i x_{\beta} F_{\alpha \nu} \\
& +2 i x_{\nu} F_{\alpha \beta}+i r^{2}\left[\nabla_{\nu}, F_{\alpha \beta}\right] \\
& =-i\left(\pi_{\alpha} \delta_{\beta \nu}-\pi_{\beta} \delta_{\alpha \nu}\right) \text {. } \\
& {\left[J_{\alpha \beta}, F_{\alpha^{\prime} \beta^{\prime}}\right]=\left[x_{\alpha} \pi_{\beta}-x_{\beta} \pi_{\alpha}+r^{2} F_{\alpha \beta}, F_{\alpha^{\prime} \beta^{\prime}}\right]} \\
& =x_{\alpha}\left[\pi_{\beta}, F_{\alpha^{\prime} \beta^{\prime}}\right]-x_{\beta}\left[\pi_{\alpha}, F_{\alpha^{\prime} \beta^{\prime}}\right]+r^{2}\left[F_{\alpha \beta}, F_{\alpha^{\prime} \beta^{\prime}}\right] \\
& =i \frac{x_{\alpha}}{r^{2}}\left(2 x_{\beta} F_{\alpha^{\prime} \beta^{\prime}}+x_{\alpha^{\prime}} F_{\beta \beta^{\prime}}+x_{\beta^{\prime}} F_{\alpha^{\prime} \beta}\right)-i \frac{x_{\beta}}{r^{2}}\left(2 x_{\alpha} F_{\alpha^{\prime} \beta^{\prime}}+x_{\alpha^{\prime}} F_{\alpha \beta^{\prime}}+x_{\beta^{\prime}} F_{\alpha^{\prime} \alpha}\right) \\
& +r^{2}\left[F_{\alpha \beta}, F_{\alpha^{\prime} \beta^{\prime}}\right] \\
& =r^{2}\left[F_{\alpha \beta}, F_{\alpha^{\prime} \beta^{\prime}}\right]-\frac{i}{r^{2}}\left(-x_{\alpha} x_{\alpha^{\prime}} F_{\beta \beta^{\prime}}-x_{\alpha} x_{\beta^{\prime}} F_{\alpha^{\prime} \beta}+x_{\beta} x_{\alpha^{\prime}} F_{\alpha \beta^{\prime}}+x_{\beta} x_{\beta^{\prime}} F_{\alpha^{\prime} \alpha}\right) \\
& =i \delta_{\alpha \alpha^{\prime}} F_{\beta \beta^{\prime}}+i \delta_{\beta \beta^{\prime}} F_{\alpha \alpha^{\prime}}-i \delta_{\alpha \beta^{\prime}} F_{\beta \alpha^{\prime}}-i \delta_{\beta \alpha^{\prime}} F_{\alpha \beta^{\prime}} \text {. }
\end{aligned}
$$

By using Lemma 2 and the definition of $J_{\alpha \beta}$, one can easily check that $J_{\alpha \beta}$ 's satisfy the standard commutation relation of $\mathfrak{s o}(D)$ Lie algebra. Then Lemma 2 may be paraphrased as follows: under the commutation action of $J_{\alpha \beta}$ 's, $r$ and $\frac{1}{r}$ transform as $\mathfrak{s o}(D)$ scalars, $x_{\alpha}$ 's and $\pi_{\alpha}$ 's transform as $\mathfrak{s o}(D)$ vectors, and $F_{\alpha \beta}$ 's transform as a $\mathfrak{s o}(D)$ bi-vectors. It is then clear that $T, \Gamma_{D+1}$ and $\Gamma_{-1}$ transform as $\mathfrak{s o}(D)$ scalars; $\vec{A}, \vec{M}$ and $\vec{\Gamma}$ transform as $\mathfrak{s o}(D)$ vectors. This completes the proof for Eq. (13) in the case when it involves $J_{\alpha \beta}$.

By using identities $x_{\alpha} \mathcal{A}_{\alpha}=0$ and $x_{\alpha} F_{\alpha \beta}=0$, one can check that $[-\vec{r} \cdot \nabla, \vec{r}]=-\vec{r},[-\vec{r} \cdot \nabla, r]=-r,\left[-\vec{r} \cdot \nabla, \frac{1}{r}\right]=\frac{1}{r}$, $[-\vec{r} \cdot \nabla, \vec{\pi}]=\vec{\pi}$. That is, $-\vec{r} \cdot \nabla$ is the dimension operator in physics. It is then clear that

$$
\left[\Gamma_{-1}, T\right]=-i \Gamma_{D+1}, \quad\left[\Gamma_{D+1}, T\right]=-i \Gamma_{-1}, \quad[\vec{\Gamma}, T]=\overrightarrow{0}
$$

Consequently,

$$
\left[M_{\alpha}, T\right]=\left[i\left[\Gamma_{\alpha}, \Gamma_{-1}\right], T\right]=i\left[\Gamma_{\alpha},\left[\Gamma_{-1}, T\right]\right]+i\left[\left[\Gamma_{\alpha}, T\right], \Gamma_{-1}\right]
$$




$$
\begin{aligned}
& =\left[\Gamma_{\alpha}, \Gamma_{D+1}\right]=-i A_{\alpha} \\
{\left[A_{\alpha}, T\right] } & =\left[i\left[\Gamma_{\alpha}, \Gamma_{D+1}\right], T\right]=i\left[\Gamma_{\alpha},\left[\Gamma_{D+1}, T\right]\right]+i\left[\left[\Gamma_{\alpha}, T\right], \Gamma_{D+1}\right] \\
& =\left[\Gamma_{\alpha}, \Gamma_{-1}\right]=-i M_{\alpha}
\end{aligned}
$$

This completes the proof of Eq. (13) in the case when it involves $T$.

The remaining verifications are divided into four cases.

$\underline{\text { Case } 1 .}$

$$
\begin{gathered}
{\left[\Gamma_{\alpha}, \Gamma_{\beta}\right]=-i J_{\alpha \beta}, \quad\left[\Gamma_{\alpha}, \Gamma_{D+1}\right]=-i A_{\alpha}} \\
{\left[\Gamma_{\alpha}, \Gamma_{-1}\right]=-i M_{\alpha}, \quad\left[\Gamma_{D+1}, \Gamma_{-1}\right]=-i T}
\end{gathered}
$$

which are just the defining relations. So case 1 is done.

\section{Case 2.}

$$
\left[M_{\alpha}, \Gamma_{\beta}\right]=-i \eta_{\alpha \beta} \Gamma_{-1}, \quad\left[A_{\alpha}, \Gamma_{\beta}\right]=-i \eta_{\alpha \beta} \Gamma_{D+1}
$$

or equivalently

$$
\left[Z_{\alpha}, \Gamma_{\beta}\right]=-i \eta_{\alpha \beta} X, \quad\left[W_{\alpha}, \Gamma_{\beta}\right]=-i \eta_{\alpha \beta} Y
$$

Proof. Since $\left[W_{\alpha}, \Gamma_{\beta}\right]=\left[x_{\alpha}, r \pi_{\beta}\right]=i r \delta_{\alpha \beta}=-i \eta_{\alpha \beta} Y$, we just need to verify the first identity. Note that $\left[Z_{\alpha}, r\right]=2 i \Gamma_{\alpha}$ (see case 3 below), so

$$
\begin{aligned}
{\left[Z_{\alpha}, \Gamma_{\beta}\right]=} & r\left[Z_{\alpha}, \pi_{\beta}\right]+2 i \Gamma_{\alpha} \pi_{\beta} \\
= & r\left[x_{\alpha} \pi^{2}-2 \pi_{\alpha}(\vec{r} \cdot \vec{\pi})+2 r^{2} F_{\alpha \beta} \pi_{\beta}-\frac{c}{r^{2}} x_{\alpha}+i(D-3) \pi_{\alpha}, \pi_{\beta}\right]+2 i r \pi_{\alpha} \pi_{\beta} \\
= & r\left(i \delta_{\alpha \beta} \pi^{2}-2 i x_{\alpha} F_{\gamma \beta} \pi_{\gamma}\right)+r\left(2 i F_{\alpha \beta}(\vec{r} \cdot \vec{\pi})-2 i \pi_{\alpha} \pi_{\beta}\right)+r\left(-2 i r^{2} F_{\alpha \gamma} F_{\gamma \beta}+\left[2 r^{2} F_{\alpha \gamma}, \pi_{\beta}\right] \pi_{\gamma}\right) \\
& +c r\left[\pi_{\beta}, \frac{x_{\alpha}}{r^{2}}\right]+(D-3) r F_{\alpha \beta}+2 i r \pi_{\alpha} \pi_{\beta} \\
= & i \delta_{\alpha \beta} r \pi^{2}-2 i r x_{\alpha} F_{\gamma \beta} \pi_{\gamma}+2 i r F_{\alpha \beta}(\vec{r} \cdot \vec{\pi})+r\left(-2 i r^{2} F_{\alpha \gamma} F_{\gamma \beta}+4 i x_{\beta} F_{\alpha \gamma} \pi_{\gamma}-2 r^{2}\left[\pi_{\beta}, F_{\alpha \gamma}\right] \pi_{\gamma}\right) \\
& +c r\left[\pi_{\beta}, \frac{x_{\alpha}}{r^{2}}\right]+(D-3) r F_{\alpha \beta} \\
= & i \delta_{\alpha \beta} r \pi^{2}-2 i r^{3} F_{\alpha \gamma} F_{\gamma \beta}+2 i r\left(2 x_{\beta} F_{\alpha \gamma}+x_{\alpha} F_{\beta \gamma}+x_{\gamma} F_{\alpha \beta}+r^{2}\left[\nabla_{\beta}, F_{\alpha \gamma}\right]\right) \pi_{\gamma} \\
& +c r\left[\pi_{\beta}, \frac{x_{\alpha}}{r^{2}}\right]+(D-3) r F_{\alpha \beta} \\
= & i \delta_{\alpha \beta} r \pi^{2}+2 i r^{3} F_{\gamma \alpha} F_{\gamma \beta}-i c r\left(\delta_{\alpha \beta}-2 \frac{x_{\alpha} x_{\beta}}{r^{4}}\right)+2(n-1) r F_{\alpha \beta} \\
= & i \delta_{\alpha \beta}\left(r \pi^{2}+\frac{c}{r^{2}}\right)=-i \eta_{\alpha \beta} X .
\end{aligned}
$$

\section{$\underline{\text { Case } 3 .}$}

$$
\begin{gathered}
{\left[M_{\alpha}, \Gamma_{-1}\right]=i \Gamma_{\alpha}, \quad\left[M_{\alpha}, \Gamma_{D+1}\right]=0} \\
{\left[A_{\alpha}, \Gamma_{-1}\right]=0, \quad\left[A_{\alpha}, \Gamma_{D+1}\right]=-i \Gamma_{\alpha} .}
\end{gathered}
$$


or equivalently,

$$
[\vec{W}, Y]=[\vec{Z}, X]=0, \quad[\vec{W}, X]=[\vec{Z}, Y]=2 i \vec{\Gamma}
$$

Proof. It is clear that $[\vec{W}, Y]=0$. Now $\left[W_{\alpha}, X\right]=r\left[x_{\alpha}, \pi^{2}\right]=i r\left\{\pi_{\beta}, \delta_{\alpha \beta}\right\}=2 i \Gamma_{\alpha}$. Next, using the identity $F_{\alpha \beta} x_{\beta}=0$, we have

$$
\begin{aligned}
{\left[Z_{\alpha}, Y\right] } & =\left[x_{\alpha} \pi^{2}-2 \pi_{\alpha}(\vec{r} \cdot \vec{\pi})+2 r^{2} F_{\alpha \beta} \pi_{\beta}-\frac{c}{r^{2}} x_{\alpha}+i(D-3) \pi_{\alpha}, r\right] \\
& =x_{\alpha}\left[\pi^{2}, r\right]-2\left[\pi_{\alpha}(\vec{r} \cdot \vec{\pi}), r\right]-2 i r F_{\alpha \beta} x_{\beta}+(D-3) \frac{x_{\alpha}}{r} \\
& =-i x_{\alpha}\left\{\pi_{\beta}, \frac{x_{\beta}}{r}\right\}-2 \pi_{\alpha}[(\vec{r} \cdot \vec{\pi}), r]-2\left[\pi_{\alpha}, r\right](\vec{r} \cdot \vec{\pi})+(D-3) \frac{x_{\alpha}}{r} \\
& =-(D-1) \frac{x_{\alpha}}{r}-2 i \frac{x_{\alpha}}{r} \vec{r} \cdot \vec{\pi}+2 i \pi_{\alpha} r+2 i \frac{x_{\alpha}}{r}(\vec{r} \cdot \vec{\pi})+(D-3) \frac{x_{\alpha}}{r} \\
& =-2 \frac{x_{\alpha}}{r}+2 i \pi_{\alpha} r=2 i r \pi_{\alpha}=2 i \Gamma_{\alpha} .
\end{aligned}
$$

Finally,

$$
\begin{aligned}
& {\left[\frac{1}{r}, Z_{\alpha}\right]=\left[\frac{1}{r}, x_{\alpha} \pi^{2}-2 \pi_{\alpha}(\vec{r} \cdot \vec{\pi})+2 r^{2} F_{\alpha \beta} \pi_{\beta}-\frac{c}{r^{2}} x_{\alpha}+i(D-3) \pi_{\alpha}\right]} \\
& =x_{\alpha}\left[\frac{1}{r}, \pi^{2}\right]-2\left[\frac{1}{r}, \pi_{\alpha}(\vec{r} \cdot \vec{\pi})\right]+2 r^{2} F_{\alpha \beta}\left[\frac{1}{r}, \pi_{\beta}\right]+i(D-3)\left[\frac{1}{r}, \pi_{\alpha}\right] \\
& =-i x_{\alpha}\left\{\frac{x_{\beta}}{r^{3}}, \pi_{\beta}\right\}-2 \pi_{\alpha}\left[\frac{1}{r},(\vec{r} \cdot \vec{\pi})\right]-2\left[\frac{1}{r}, \pi_{\alpha}\right](\vec{r} \cdot \vec{\pi})-2 i r^{2} F_{\alpha \beta} \frac{x_{\beta}}{r^{3}}+(D-3) \frac{x_{\alpha}}{r^{3}} \\
& =-i x_{\alpha}\left[\pi_{\beta}, \frac{x_{\beta}}{r^{3}}\right]-2 i \frac{x_{\alpha}}{r^{3}} \vec{r} \cdot \vec{\pi}+2 i \pi_{\alpha} \frac{1}{r}+2 i \frac{x_{\alpha}}{r^{3}}(\vec{r} \cdot \vec{\pi})+(D-3) \frac{x_{\alpha}}{r^{3}} \\
& =2 i \pi_{\alpha} \frac{1}{r} \\
& {\left[r \pi^{2}, Z_{\alpha}\right]=\left[r, Z_{\alpha}\right] \pi^{2}+r\left[\pi^{2}, Z_{\alpha}\right]} \\
& =-2 i \Gamma_{\alpha} \pi^{2}+r\left[\pi^{2}, Z_{\alpha}\right] \\
& =-2 i \Gamma_{\alpha} \pi^{2}+r\left[\pi^{2}, x_{\alpha} \pi^{2}-2 \pi_{\alpha}(\vec{r} \cdot \vec{\pi})+2 r^{2} F_{\alpha \beta} \pi_{\beta}-\frac{c}{r^{2}} x_{\alpha}+i(D-3) \pi_{\alpha}\right] \\
& =-2 i \Gamma_{\alpha} \pi^{2}+r\left(\left[\pi^{2}, x_{\alpha}\right] \pi^{2}-2\left[\pi^{2}, \pi_{\alpha}(\vec{r} \cdot \vec{\pi})\right]+2\left[\pi^{2}, r^{2} F_{\alpha \beta} \pi_{\beta}\right]\right) \\
& +r\left(-c\left[\pi^{2}, \frac{x_{\alpha}}{r^{2}}\right]+i(D-3)\left[\pi^{2}, \pi_{\alpha}\right]\right) \\
& =-2 i \Gamma_{\alpha} \pi^{2}+r\left(-2 i \pi_{\alpha} \pi^{2}-2\left[\pi^{2}, \pi_{\alpha}\right](\vec{r} \cdot \vec{\pi})+4 i \pi_{\alpha} \pi^{2}+2\left[\pi^{2}, r^{2} F_{\alpha \beta} \pi_{\beta}\right]\right) \\
& +r\left(-c\left[\pi^{2}, \frac{x_{\alpha}}{r^{2}}\right]+i(D-3)\left[\pi^{2}, \pi_{\alpha}\right]\right) \\
& =r\left(-2\left[\pi^{2}, \pi_{\alpha}\right](\vec{r} \cdot \vec{\pi})+2\left[\pi^{2}, r^{2} F_{\alpha \beta}\right] \pi_{\beta}+2 r^{2} F_{\alpha \beta}\left[\pi^{2}, \pi_{\beta}\right]-c\left[\pi^{2}, \frac{x_{\alpha}}{r^{2}}\right]+i(D-3)\left[\pi^{2}, \pi_{\alpha}\right]\right) \\
& =r\left(-2\left[\pi^{2}, \pi_{\alpha}\right](\vec{r} \cdot \vec{\pi})-2\left[\pi^{2}, x_{\alpha} \pi_{\beta}-x_{\beta} \pi_{\alpha}\right] \pi_{\beta}\right) \\
& +r\left(2 r^{2} F_{\alpha \beta}\left[\pi^{2}, \pi_{\beta}\right]-c\left[\pi^{2}, \frac{x_{\alpha}}{r^{2}}\right]+i(D-3)\left[\pi^{2}, \pi_{\alpha}\right]\right) \\
& =r\left(-2\left[\pi^{2}, \pi_{\alpha}\right](\vec{r} \cdot \vec{\pi})-2\left[\pi^{2}, x_{\alpha} \pi_{\beta}\right] \pi_{\beta}+2\left[\pi^{2}, x_{\beta} \pi_{\alpha}\right] \pi_{\beta}\right) \\
& +r\left(2 r^{2} F_{\alpha \beta}\left[\pi^{2}, \pi_{\beta}\right]-c\left[\pi^{2}, \frac{x_{\alpha}}{r^{2}}\right]+i(D-3)\left[\pi^{2}, \pi_{\alpha}\right]\right) \\
& =r\left(-2\left[\pi^{2}, \pi_{\alpha}\right](\vec{r} \cdot \vec{\pi})-2 x_{\alpha}\left[\pi^{2}, \pi_{\beta}\right] \pi_{\beta}+2 x_{\beta}\left[\pi^{2}, \pi_{\alpha}\right] \pi_{\beta}+4 F_{\alpha \beta} \pi_{\beta}\right) \\
& +r\left(2 r^{2} F_{\alpha \beta}\left[\pi^{2}, \pi_{\beta}\right]-c\left[\pi^{2}, \frac{x_{\alpha}}{r^{2}}\right]+i(D-3)\left[\pi^{2}, \pi_{\alpha}\right]\right) .
\end{aligned}
$$

To continue we note that $\left[\pi^{2}, \pi_{\alpha}\right]=2 i F_{\alpha \gamma} \pi_{\gamma}$, so

$$
\left[r \pi^{2}, Z_{\alpha}\right]=r\left(-4 i F_{\alpha \gamma} \pi_{\gamma}(\vec{r} \cdot \vec{\pi})-4 i x_{\alpha} F_{\beta \gamma} \pi_{\gamma} \pi_{\beta}+4 i x_{\beta} F_{\alpha \gamma} \pi_{\gamma} \pi_{\beta}+4 F_{\alpha \beta} \pi_{\beta}\right)
$$




$$
\begin{aligned}
& \left.+r\left(4 i r^{2} F_{\alpha \beta} F_{\beta \gamma} \pi_{\gamma}-c\left[\pi^{2}, \frac{x_{\alpha}}{r^{2}}\right]-2(D-3) F_{\alpha \gamma} \pi_{\gamma}\right]\right) \\
= & \left.r\left(2 x_{\alpha} F_{\beta \gamma} F_{\beta \gamma}+4 i r^{2} F_{\alpha \beta} F_{\beta \gamma} \pi_{\gamma}-c\left[\pi^{2}, \frac{x_{\alpha}}{r^{2}}\right]-2(D-3) F_{\alpha \gamma} \pi_{\gamma}\right]\right) \\
= & 4 c_{2} \frac{x_{\alpha}}{r^{3}}-\operatorname{cr}\left[\pi^{2}, \frac{x_{\alpha}}{r^{2}}\right]+4 i r\left(r^{2} F_{\alpha \beta} F_{\beta \gamma}+i \frac{D-3}{2} F_{\alpha \gamma}\right) \pi_{\gamma} \\
= & 4 c_{2} \frac{x_{\alpha}}{r^{3}}+i c r\left\{\pi_{\beta},\left[\nabla_{\beta}, \frac{x_{\alpha}}{r^{2}}\right]\right\}-4 i r \frac{c_{2}}{n}\left(\frac{\delta_{\alpha \gamma}}{r^{2}}-\frac{x_{\alpha} x_{\gamma}}{r^{4}}\right) \pi_{\gamma} \\
= & 4 c_{2} \frac{x_{\alpha}}{r^{3}}+i c r\left\{\pi_{\beta}, \frac{\delta_{\alpha \beta}}{r^{2}}-2 \frac{x_{\alpha} x_{\beta}}{r^{4}}\right\}+4 i r c\left(-\frac{1}{r^{2}} \pi_{\alpha}+\frac{x_{\alpha}}{r^{4}} \vec{r} \cdot \vec{\pi}\right) \\
= & 4 c_{2} \frac{x_{\alpha}}{r^{3}}+i c r\left[\pi_{\beta}, \frac{\delta_{\alpha \beta}}{r^{2}}-2 \frac{x_{\alpha} x_{\beta}}{r^{4}}\right]-2 i c \frac{1}{r} \pi_{\alpha} .
\end{aligned}
$$

Therefore,

$$
\begin{aligned}
{\left[X, Z_{\alpha}\right] } & =\left[r \pi^{2}+\frac{c}{r}, Z_{\alpha}\right]=4 c_{2} \frac{x_{\alpha}}{r^{3}}+i c r\left[\pi_{\beta}, \frac{\delta_{\alpha \beta}}{r^{2}}-2 \frac{x_{\alpha} x_{\beta}}{r^{4}}\right]-2 i c\left[\frac{1}{r}, \pi_{\alpha}\right] \\
& =4 n c \frac{x_{\alpha}}{r^{3}}-2 c(D-2) \frac{x_{\alpha}}{r^{3}}-2 c \frac{x_{\alpha}}{r^{3}}=0 .
\end{aligned}
$$

$\underline{\text { Case } 4 .}$

$$
\left[M_{\alpha}, M_{\beta}\right]=-i J_{\alpha \beta}, \quad\left[A_{\alpha}, M_{\beta}\right]=-i \eta_{\alpha \beta} T, \quad\left[A_{\alpha}, A_{\beta}\right]=i J_{\alpha \beta}
$$

or equivalently,

$$
\left[Z_{\alpha}, Z_{\beta}\right]=\left[W_{\alpha}, W_{\beta}\right]=0, \quad\left[Z_{\alpha}, W_{\beta}\right]=-2 i\left(\eta_{\alpha \beta} T+J_{\alpha \beta}\right)
$$

Proof. It is clear that $\left[W_{\alpha}, W_{\beta}\right]=0$ because $W_{\alpha}=x_{\alpha}$. Next,

$$
\begin{aligned}
{\left[Z_{\alpha}, W_{\beta}\right] } & =\left[x_{\alpha} \pi^{2}-2 \pi_{\alpha}(\vec{r} \cdot \vec{\pi})+2 r^{2} F_{\alpha \gamma} \pi_{\gamma}-\frac{c}{r^{2}} x_{\alpha}+i(D-3) \pi_{\alpha}, x_{\beta}\right] \\
& =x_{\alpha}\left[\pi^{2}, x_{\beta}\right]-2\left[\pi_{\alpha}(\vec{r} \cdot \vec{\pi}), x_{\beta}\right]+2 r^{2} F_{\alpha \gamma}\left[\pi_{\gamma}, x_{\beta}\right]+i(D-3)\left[\pi_{\alpha}, x_{\beta}\right] \\
& =-2 i x_{\alpha} \pi_{\beta}-2 \pi_{\alpha}\left[(\vec{r} \cdot \vec{\pi}), x_{\beta}\right]-2\left[\pi_{\alpha}, x_{\beta}\right](\vec{r} \cdot \vec{\pi})-2 i r^{2} F_{\alpha \beta}+(D-3) \delta_{\alpha \beta} \\
& =-2 i x_{\alpha} \pi_{\beta}+2 i \pi_{\alpha} x_{\beta}+2 i \delta_{\alpha \beta}(\vec{r} \cdot \vec{\pi})-2 i r^{2} F_{\alpha \beta}+(D-3) \delta_{\alpha \beta} \\
& =-2 i\left(x_{\alpha} \pi_{\beta}-x_{\beta} \pi_{\alpha}+r^{2} F_{\alpha \beta}\right)+2 i \delta_{\alpha \beta}\left(\vec{r} \cdot \vec{\pi}-i \frac{D-1}{2}\right) \\
& =-2 i\left(\eta_{\alpha \beta} T+J_{\alpha \beta}\right) .
\end{aligned}
$$

Finally, using results from case 2 and case 3, we have

$$
\begin{aligned}
-i\left[Z_{\alpha}, Z_{\beta}\right] & =\left[\left[\Gamma_{\alpha}, X\right], Z_{\beta}\right]=\left[\Gamma_{\alpha} X-X \Gamma_{\alpha}, Z_{\beta}\right] \\
& =\left[\Gamma_{\alpha} X, Z_{\beta}\right]-\left[X \Gamma_{\alpha}, Z_{\beta}\right]=\left[\Gamma_{\alpha}, Z_{\beta}\right] X-X\left[\Gamma_{\alpha}, Z_{\beta}\right] \\
& =\left[\left[\Gamma_{\alpha}, Z_{\beta}\right], X\right]=\left[i \eta_{\alpha \beta} X, X\right]=0 .
\end{aligned}
$$

End of the proof of part 1) of Theorem 2 . 


\section{B. Proof of part 2)}

We just need to verify equality

$$
\sum_{1 \leq A \leq D+1}\left\{J_{A B}, J_{A C}\right\}-\sum_{-1 \leq A \leq 0}\left\{J_{A B}, J_{A C}\right\}=2 a \eta_{B C}
$$

under the condition that $B \leq C$, to be more specific, we need to verify the following identities:

$$
\left\{\begin{aligned}
\sum_{1 \leq \alpha \leq D}\left\{J_{\alpha \beta}, J_{\alpha \gamma}\right\}+\left\{A_{\beta}, A_{\gamma}\right\}-\left\{M_{\beta}, M_{\gamma}\right\}-\left\{\Gamma_{\beta}, \Gamma_{\gamma}\right\} & =2 a \eta_{\beta \gamma} \\
\sum_{1 \leq \alpha \leq D}\left\{J_{\alpha \beta}, A_{\alpha}\right\}-\left\{M_{\beta}, T\right\}-\left\{\Gamma_{\beta}, \Gamma_{D+1}\right\} & =0 \\
\sum_{1 \leq \alpha \leq D} A_{\alpha}^{2}-T^{2}-\Gamma_{D+1}^{2} & =-a \\
\sum_{1 \leq \alpha \leq D}\left\{J_{\alpha \beta}, M_{\alpha}\right\}-\left\{A_{\beta}, T\right\}-\left\{\Gamma_{\beta}, \Gamma_{-1}\right\} & =0 \\
\sum_{1 \leq \alpha \leq D}\left\{A_{\alpha}, M_{\alpha}\right\}-\left\{\Gamma_{D+1}, \Gamma_{-1}\right\} & =0 \\
\sum_{1 \leq \alpha \leq D} M_{\alpha}^{2}+T^{2}-\Gamma_{-1}^{2} & =a \\
\sum_{1 \leq \alpha \leq D}\left\{J_{\alpha \beta}, \Gamma_{\alpha}\right\}-\left\{A_{\beta}, \Gamma_{D+1}\right\}+\left\{M_{\beta}, \Gamma_{-1}\right\} & =0 \\
\sum_{1 \leq \alpha \leq D}\left\{A_{\alpha}, \Gamma_{\alpha}\right\}+\left\{T, \Gamma_{-1}\right\} & =0 \\
\sum_{1 \leq \alpha \leq D}\left\{M_{\alpha}, \Gamma_{\alpha}\right\}+\left\{\Gamma_{D+1}, T\right\} & =0 \\
\sum_{1 \leq \alpha \leq D} \Gamma_{\alpha}^{2}+\Gamma_{D+1}^{2}-\Gamma_{-1}^{2} & =a
\end{aligned}\right.
$$

The checking is then divided into six cases.

\section{Case 1.}

$$
\sum_{1 \leq \alpha \leq D} \Gamma_{\alpha}^{2}+\Gamma_{D+1}^{2}-\Gamma_{-1}^{2}=a
$$

Proof.

$$
\begin{aligned}
\sum_{1 \leq \alpha \leq D} \Gamma_{\alpha}^{2}+\Gamma_{D+1}^{2}-\Gamma_{-1}^{2} & =r \pi_{\alpha} r \pi_{\alpha}-\frac{1}{2}(X Y+Y X) \\
& =r^{2} \pi^{2}-i \vec{r} \cdot \vec{\pi}-\frac{1}{2}\left(r \pi^{2} r+c+r^{2} \pi^{2}+c\right) \\
& =-i \vec{r} \cdot \vec{\pi}-\frac{1}{2} r\left[\pi^{2}, r\right]-c=-i \vec{r} \cdot \vec{\pi}+\frac{i}{2} r\left\{\pi_{\mu}, \frac{x_{\mu}}{r}\right\}-c \\
& =\frac{i}{2} r\left[\pi_{\mu}, \frac{x_{\mu}}{r}\right]-c=\frac{D-1}{2}-c=a .
\end{aligned}
$$

\section{Case 2.}

$$
\sum_{1 \leq \alpha \leq D}\left\{A_{\alpha}, \Gamma_{\alpha}\right\}+\left\{T, \Gamma_{-1}\right\}=0, \quad \sum_{1 \leq \alpha \leq D}\left\{M_{\alpha}, \Gamma_{\alpha}\right\}+\left\{\Gamma_{D+1}, T\right\}=0
$$

Or equivalently

$$
\sum_{1 \leq \alpha \leq D}\left\{Z_{\alpha}, \Gamma_{\alpha}\right\}+\{X, T\}=0, \quad \sum_{1 \leq \alpha \leq D}\left\{W_{\alpha}, \Gamma_{\alpha}\right\}-\{Y, T\}=0
$$


Proof. We check the 2nd identity first:

$$
\begin{aligned}
\sum_{1 \leq \alpha \leq D}\left\{W_{\alpha}, \Gamma_{\alpha}\right\}-\{Y, T\} & =\left\{x_{\alpha}, r \pi_{\alpha}\right\}-\left\{r, \vec{r} \cdot \vec{\pi}-\frac{D-1}{2} i\right\} \\
& =2 r \vec{r} \cdot \vec{\pi}+r\left[\pi_{\alpha}, x_{\alpha}\right]-2 r \vec{r} \cdot \vec{\pi}-[\vec{r} \cdot \vec{\pi}, r]+i(D-1) r \\
& =0 .
\end{aligned}
$$

Then we check the 1st identity:

$$
\begin{aligned}
\sum_{1 \leq \alpha \leq D}\left\{Z_{\alpha}, \Gamma_{\alpha}\right\}+\{X, T\}= & 2 \Gamma_{\alpha} Z_{\alpha}+2 T X+\left[Z_{\alpha}, \Gamma_{\alpha}\right]+[X, T] \\
= & 2\left(r \pi_{\alpha} x_{\alpha} \pi^{2}-2 r \pi^{2}(\vec{r} \cdot \vec{\pi})+2 r \pi_{\alpha} r^{2} F_{\alpha \beta} \pi_{\beta}-r \pi_{\alpha} \frac{c}{r^{2}} x_{\alpha}+i(D-3) r \pi^{2}\right) \\
& +2\left(\vec{r} \cdot \vec{\pi}-\frac{D-1}{2} i\right)\left(r \pi^{2}+\frac{c}{r}\right)-i \eta_{\alpha \alpha} X-i X \\
= & 2\left(r(\vec{r} \cdot \pi) \pi^{2}-2 r \pi^{2}(\vec{r} \cdot \vec{\pi})+2 r^{3} F_{\alpha \beta} \pi_{\alpha} \pi_{\beta}-c r \pi_{\alpha} \frac{x_{\alpha}}{r^{2}}-3 i r \pi^{2}\right) \\
& +2 \vec{r} \cdot \vec{\pi}\left(r \pi^{2}+\frac{c}{r}\right) \\
= & 2\left(2 i r \pi^{2}-r \pi^{2}(\vec{r} \cdot \vec{\pi})-i r^{3} F_{\alpha \beta} F_{\alpha \beta}-\frac{c}{r} \vec{r} \cdot \vec{\pi}-c r\left[\pi_{\alpha}, \frac{x_{\alpha}}{r^{2}}\right]-3 i r \pi^{2}\right) \\
& +2 \vec{r} \cdot \vec{\pi}\left(r \pi^{2}+\frac{c}{r}\right) \\
= & 2\left(\left[\vec{r} \cdot \vec{\pi}, r \pi^{2}\right]-i r^{3} F_{\alpha \beta} F_{\alpha \beta}+\left[\vec{r} \cdot \vec{\pi}, \frac{c}{r}\right]-c r\left[\pi_{\alpha}, \frac{x_{\alpha}}{r^{2}}\right]-i r \pi^{2}\right) \\
= & 2\left(-i r^{3} F_{\alpha \beta} F_{\alpha \beta}+i \frac{c}{r}+i c \frac{D-2}{r}\right) \\
= & 2\left(-2 i \frac{c_{2}}{r}+i c \frac{D-1}{r}\right)=0 .
\end{aligned}
$$

$\underline{\text { Case } 3 .}$

$$
\sum_{1 \leq \alpha \leq D}\left\{J_{\alpha \beta}, \Gamma_{\alpha}\right\}-\left\{A_{\beta}, \Gamma_{D+1}\right\}+\left\{M_{\beta}, \Gamma_{-1}\right\}=0 .
$$

Proof.

$$
\begin{aligned}
& \sum_{1 \leq \alpha \leq D}\left\{J_{\alpha \beta}, \Gamma_{\alpha}\right\}-\left\{A_{\beta}, \Gamma_{D+1}\right\}+\left\{M_{\beta}, \Gamma_{-1}\right\} \\
= & 2 J_{\alpha \beta} \Gamma_{\alpha}+\left[\Gamma_{\alpha}, J_{\alpha \beta}\right]+\frac{1}{2}\left(\left\{X, W_{\beta}\right\}+\left\{Y, Z_{\beta}\right\}\right) \\
= & 2\left(x_{\alpha} \pi_{\beta} r \pi_{\alpha}-x_{\beta} \pi_{\alpha} r \pi_{\alpha}+r^{2} F_{\alpha \beta} r \pi_{\alpha}\right)-i(D-1) \Gamma_{\beta} \\
& +X W_{\beta}+Y Z_{\beta}+2 i \Gamma_{\beta} \\
= & 2\left(\pi_{\beta} r \vec{r} \cdot \vec{\pi}+\left[x_{\alpha}, \pi_{\beta} r\right] \pi_{\alpha}-x_{\beta}\left[\pi_{\alpha}, r\right] \pi_{\alpha}-x_{\beta} r \pi^{2}+r^{3} F_{\alpha \beta} \pi_{\alpha}\right)-i(D-3) \Gamma_{\beta} \\
& +\left(r \pi^{2}+\frac{c}{r}\right) x_{\beta}+r x_{\beta} \pi^{2}-2 r \pi_{\beta}(\vec{r} \cdot \vec{\pi})+2 r^{3} F_{\beta \gamma} \pi_{\gamma}-\frac{c}{r} x_{\beta}+i(D-3) r \pi_{\beta} \\
= & 2\left(\pi_{\beta} r \vec{r} \cdot \vec{\pi}+i r \pi_{\beta}+i \frac{x_{\beta}}{r} \vec{r} \cdot \pi\right)-x_{\beta} r \pi^{2} \\
& +r \pi^{2} x_{\beta}-2 r \pi_{\beta}(\vec{r} \cdot \vec{\pi}) \\
= & 2\left(r \pi_{\beta} \vec{r} \cdot \vec{\pi}+i r \pi_{\beta}\right)+\left[r \pi^{2}, x_{\beta}\right]-2 r \pi_{\beta}(\vec{r} \cdot \vec{\pi}) \\
= & 2\left(\left[r \pi_{\beta}, \vec{r} \cdot \vec{\pi}\right]+i r \pi_{\beta}\right)-2 i r \pi_{\beta}=0 .
\end{aligned}
$$


Case 4.

$$
\begin{aligned}
\sum_{1 \leq \alpha \leq D} A_{\alpha}^{2}-T^{2}-\Gamma_{D+1}^{2} & =-a \\
\sum_{1 \leq \alpha \leq D} M_{\alpha}^{2}+T^{2}-\Gamma_{-1}^{2} & =a \\
\sum_{1 \leq \alpha \leq D}\left\{A_{\alpha}, M_{\alpha}\right\}-\left\{\Gamma_{D+1}, \Gamma_{-1}\right\} & =0
\end{aligned}
$$

or equivalently

$$
\sum_{1 \leq \alpha \leq D} Z_{\alpha}^{2}=X^{2}, \quad \sum_{1 \leq \alpha \leq D}\left\{Z_{\alpha}, W_{\alpha}\right\}+4 T^{2}-\{X, Y\}=4 a
$$

Here we have used the fact that $\sum_{1 \leq \alpha \leq D} W_{\alpha}^{2}=Y^{2}$.

Proof. To check the 1 st identity, we note that $Z_{\alpha}=i\left[\Gamma_{\alpha}, X\right]$ and $\left[Z_{\alpha}, X\right]=0$, so

$$
Z_{\alpha}^{2}=\frac{i}{2}\left[\left\{Z_{\alpha}, \Gamma_{\alpha}\right\}, X\right]
$$

Then

$$
\begin{aligned}
\sum_{1 \leq \alpha \leq D} Z_{\alpha}^{2} & =\frac{i}{2} \sum_{1 \leq \alpha \leq D}\left[\left\{Z_{\alpha}, \Gamma_{\alpha}\right\}, X\right] \\
& =-\frac{i}{2}[\{X, T\}, X] \quad \text { use results from case } 2 \\
& =-\frac{i}{2}\left[T, X^{2}\right]=X^{2} .
\end{aligned}
$$

To check the 2 nd identity, we note that $Z_{\alpha}=i\left[\Gamma_{\alpha}, X\right]$ and $\left[W_{\alpha}, X\right]=2 i \Gamma_{\alpha}$, so

$$
\left\{Z_{\alpha}, W_{\alpha}\right\}=i\left[\left\{W_{\alpha}, \Gamma_{\alpha}\right\}, X\right]+4 \Gamma_{\alpha}^{2}
$$

Then

$$
\begin{aligned}
\sum_{1 \leq \alpha \leq D}\left\{Z_{\alpha}, W_{\alpha}\right\} & =i[\{Y, T\}, X]+4 \sum_{1 \leq \alpha \leq D} \Gamma_{\alpha}^{2} \quad \text { use results from case } 2 \\
& =i(\{Y,[T, X]\}+\{[Y, X], T\})+4 \sum_{1 \leq \alpha \leq D} \Gamma_{\alpha}^{2} \\
& =i(\{Y, i X\}+\{2 i T, T\})+4 \sum_{1 \leq \alpha \leq D} \Gamma_{\alpha}^{2} \\
& =-\{X, Y\}-4 T^{2}+4 \sum_{1 \leq \alpha \leq D} \Gamma_{\alpha}^{2}
\end{aligned}
$$

So

$$
\begin{aligned}
\sum_{1 \leq \alpha \leq D}\left\{Z_{\alpha}, W_{\alpha}\right\}+4 T^{2}-\{X, Y\} & =4 \sum_{1 \leq \alpha \leq D} \Gamma_{\alpha}^{2}-2\{X, Y\} \\
& =4 a^{\text {use results from case } 1 .}
\end{aligned}
$$


$\underline{\text { Case } 5 .}$.

$$
\begin{aligned}
& \sum_{1 \leq \alpha \leq D}\left\{J_{\alpha \beta}, A_{\alpha}\right\}-\left\{M_{\beta}, T\right\}-\left\{\Gamma_{\beta}, \Gamma_{D+1}\right\}=0 \\
& \sum_{1 \leq \alpha \leq D}\left\{J_{\alpha \beta}, M_{\alpha}\right\}-\left\{A_{\beta}, T\right\}-\left\{\Gamma_{\beta}, \Gamma_{-1}\right\}=0
\end{aligned}
$$

or equivalently

$$
\left\{\begin{array}{c}
\sum_{1 \leq \alpha \leq D}\left\{J_{\alpha \beta}, Z_{\alpha}\right\}-\left\{Z_{\beta}, T\right\}-\left\{\Gamma_{\beta}, X\right\}=0 \\
\sum_{1 \leq \alpha \leq D}\left\{J_{\alpha \beta}, W_{\alpha}\right\}+\left\{W_{\beta}, T\right\}-\left\{\Gamma_{\beta}, Y\right\}=0 .
\end{array}\right.
$$

Proof. We check the 2nd identity first:

$$
\begin{aligned}
& \sum_{1 \leq \alpha \leq D}\left\{J_{\alpha \beta}, W_{\alpha}\right\}+\left\{W_{\beta}, T\right\}-\left\{\Gamma_{\beta}, Y\right\} \\
= & 2 x_{\alpha} J_{\alpha \beta}+\left[J_{\alpha \beta}, x_{\alpha}\right]+2 x_{\beta} \vec{r} \cdot \vec{\pi}+\left[\vec{r} \cdot \vec{\pi}, x_{\beta}\right]-i(D-1) x_{\beta}-2 r^{2} \pi_{\beta}-r\left[\pi_{\beta}, r\right] \\
= & 2 r^{2} \pi_{\beta}-2 x_{\beta} \vec{r} \cdot \vec{\pi}+\left[J_{\alpha \beta}, x_{\alpha}\right]+2 x_{\beta} \vec{r} \cdot \vec{\pi}+\left[\vec{r} \cdot \vec{\pi}, x_{\beta}\right]-i(D-1) x_{\beta}-2 r^{2} \pi_{\beta}-r\left[\pi_{\beta}, r\right]=0 .
\end{aligned}
$$

To check the 1 st identity, we note that $Z_{\alpha}=i\left[\Gamma_{\alpha}, X\right]$ and $\left[J_{\alpha \beta}, X\right]=0$, so

$$
\begin{aligned}
\sum_{1 \leq \alpha \leq D}\left\{J_{\alpha \beta}, Z_{\alpha}\right\} & =i\left[\left\{J_{\alpha \beta}, \Gamma_{\alpha}\right\}, X\right] \\
& =-i\left[X W_{\beta}+Z_{\beta} Y, X\right] \quad \text { Use results from case } 3 \\
& =-i X\left[W_{\beta}, X\right]-i\left[Z_{\beta}, X\right] Y-i Z_{\beta}[Y, X] \\
& =2 X \Gamma_{\beta}+2 Z_{\beta} T \quad \text { Use results from commutation relations } \\
& =\left\{X, \Gamma_{\beta}\right\}+\left\{Z_{\beta}, T\right\}+\left[X, \Gamma_{\beta}\right]+\left[Z_{\beta}, T\right] \\
& =\left\{X, \Gamma_{\beta}\right\}+\left\{Z_{\beta}, T\right\}+i Z_{\beta}-i Z_{\beta}=\left\{X, \Gamma_{\beta}\right\}+\left\{Z_{\beta}, T\right\} .
\end{aligned}
$$

So the 1st identity is checked.

Case 6.

$$
\sum_{1 \leq \alpha \leq D}\left\{J_{\alpha \beta}, J_{\alpha \gamma}\right\}+\left\{A_{\beta}, A_{\gamma}\right\}-\left\{M_{\beta}, M_{\gamma}\right\}-\left\{\Gamma_{\beta}, \Gamma_{\gamma}\right\}=2 a \eta_{\beta \gamma} .
$$

Proof.

$$
\begin{aligned}
\sum_{1 \leq \alpha \leq D}\left\{J_{\alpha \beta}, J_{\alpha \gamma}\right\}= & i \sum_{1 \leq \alpha \leq D}\left\{J_{\alpha \beta},\left[\Gamma_{\alpha}, \Gamma_{\gamma}\right]\right\} \\
= & i \sum_{1 \leq \alpha \leq D}\left(\left[\left\{J_{\alpha \beta}, \Gamma_{\alpha}\right\}, \Gamma_{\gamma}\right]-\left\{\left[J_{\alpha \beta}, \Gamma_{\gamma}\right], \Gamma_{\alpha}\right\}\right) \\
= & -i\left[X W_{\beta}+Z_{\beta} Y, \Gamma_{\gamma}\right]-\left\{\Gamma_{\alpha} \delta_{\beta \gamma}-\Gamma_{\beta} \delta_{\alpha \gamma}, \Gamma_{\alpha}\right\} \\
= & -i X\left[W_{\beta}, \Gamma_{\gamma}\right]-i Z_{\beta}\left[Y, \Gamma_{\gamma}\right]-i\left[X, \Gamma_{\gamma}\right] W_{\beta}-i\left[Z_{\beta}, \Gamma_{\gamma}\right] Y \quad \text { use results from case } 3 \\
& -2 \delta_{\beta \gamma} \sum_{1 \leq \alpha \leq D} \Gamma_{\alpha}^{2}+\left\{\Gamma_{\beta}, \Gamma_{\gamma}\right\} \\
= & -\eta_{\beta \gamma} Y X+Z_{\beta} W_{\gamma}+Z_{\gamma} W_{\beta}-\eta_{\beta \gamma} Y X \\
& +2 \eta_{\beta \gamma} \sum_{1 \leq \alpha \leq D} \Gamma_{\alpha}^{2}+\left\{\Gamma_{\beta}, \Gamma_{\gamma}\right\} .
\end{aligned}
$$


So

$$
\begin{aligned}
\sum_{1 \leq \alpha \leq D}\left\{J_{\alpha \beta}, J_{\alpha \gamma}\right\}-\left\{\Gamma_{\beta}, \Gamma_{\gamma}\right\}= & \eta_{\beta \gamma}\left(2 \sum_{1 \leq \alpha \leq D} \Gamma_{\alpha}^{2}-2 Y X\right)+Z_{\beta} W_{\gamma}+Z_{\gamma} W_{\beta} \\
= & \eta_{\beta \gamma}\left(2 \sum_{1 \leq \alpha \leq D} \Gamma_{\alpha}^{2}-2 Y X\right)+\frac{1}{2}\left(\left\{Z_{\beta}, W_{\gamma}\right\}+\left\{Z_{\gamma}, W_{\beta}\right\}\right) \\
& -\frac{1}{2}\left(\left[Z_{\beta}, W_{\gamma}\right]+\left[Z_{\gamma}, W_{\beta}\right]\right) \\
= & \eta_{\beta \gamma}\left(2 \sum_{1 \leq \alpha \leq D} \Gamma_{\alpha}^{2}-2 Y X+2 i T\right)+\frac{1}{2}\left(\left\{Z_{\beta}, W_{\gamma}\right\}+\left\{Z_{\gamma}, W_{\beta}\right\}\right) \\
= & \eta_{\beta \gamma}\left(2 \sum_{1 \leq \alpha \leq D} \Gamma_{\alpha}^{2}-\{X, Y\}\right)-\left\{A_{\beta}, A_{\gamma}\right\}+\left\{M_{\beta}, M_{\gamma}\right\} .
\end{aligned}
$$

So the identity is true because in case 1 we have verified that

$$
2 \sum_{1 \leq \alpha \leq D} \Gamma_{\alpha}^{2}-\{X, Y\}=2 a
$$

End of the proof of part 2) of Theorem 2 .

\section{REPRESENTATION THEORETICAL ASPECTS — THE PRELIMINARY PART}

The main objective in the rest of this paper is to show that the algebraic direct sum $\mathcal{H}$ of the energy eigenspaces of a generalized MICZ-Kepler problem in dimension $(2 n+1)$ is a unitary highest weight $(\mathfrak{g}, K)$-module where $\mathfrak{g}=\mathfrak{s o}(2 n+4)$ and $K=\operatorname{Spin}(2) \times_{\mathbb{Z}_{2}} \operatorname{Spin}(2 n+2)$. Along the way, we prove Theorem 1 .

We can label the generators of $\mathfrak{g}_{0}$ (the Lie algebra of $\operatorname{Spin}(2,2 n+2)$ ) as follows:

$$
M_{A B}=-M_{B A} \quad \text { for } A, B=-1,0,1, \ldots, 2 n+2
$$

where in the $(2 n+4)$-dimensional defining representation, the matrix elements of $M_{A B}$ are given by

$$
\left[M_{A B}\right]_{J K}=-i\left(\eta_{A J} \eta_{B K}-\eta_{B J} \eta_{A K}\right)
$$

with the indefinite metric tensor $\eta$ being $\operatorname{diag}\{++-\cdots-\}$ relative to the following order: $-1,0,1, \ldots, 2 n+2$ for the indices.

One can easily show that

$$
\left[M_{A B}, M_{A^{\prime} B^{\prime}}\right]=i\left(\eta_{A A^{\prime}} M_{B B^{\prime}}+\eta_{B B^{\prime}} M_{A A^{\prime}}-\eta_{A B^{\prime}} M_{B A^{\prime}}-\eta_{B A^{\prime}} M_{A B^{\prime}}\right) .
$$

In view of the sign difference between the right hand sides of Eqs. (13) and (30), we define the representation $\left(\tilde{\pi}, C^{\infty}\left(\mathcal{S}^{2 \mu}\right)\right)$ of $\mathfrak{g}$ as follows: for $\psi \in C^{\infty}\left(\mathcal{S}^{2 \mu}\right)$,

$$
\tilde{\pi}\left(M_{A B}\right)(\psi)=-\hat{J}_{A B} \psi
$$


where, by definition, $\hat{J}_{A B}:=\frac{1}{\sqrt{r}} J_{A B} \sqrt{r}$.

However, what is really relevant for us is just a subspace of $C^{\infty}\left(\mathcal{S}^{2 \mu}\right)$, i.e., $\mathcal{H}$. Actually, the story is bit more involved: what is really invariant under $\tilde{\pi}$ is not $\mathcal{H}$, but a twisted version of $\mathcal{H}$ which is denoted by $\tilde{\mathcal{H}}$ later; and there is a twist linear equivalence

$$
\tau: \mathcal{H} \rightarrow \tilde{\mathcal{H}}
$$

which preserves the $L^{2}$-norm, such that, viewing $\tau$ as an equivalence of representations, we get representation $(\pi, \mathcal{H})$. Because of this intricacy, we shall devote the next two subsections to some preparations.

\section{A. Review of the (bound) energy eigenspaces}

The bound eigen-states (i.e., $L^{2}$ eigen-sections of the Hamiltonian) of the generalized MICZ-Kepler problems have been analyzed in section 5.1 of Ref. [4] by using the classical analytic method with the help of the representation theory for compact Lie groups. Recall that the (bound) energy spectrum is

$$
E_{I}=-\frac{1}{2(I+n+|\mu|)^{2}}
$$

where $I=0,1,2, \cdots$.

Denote by $\left.\mathcal{S}^{2 \mu}\right|_{S^{2 n}}$ the restriction bundle of $\mathcal{S}^{2 \mu}$ to the unit sphere $S^{2 n}$. As a hermitian bundle with a hermitian connection, $\left.\mathcal{S}^{2 \mu}\right|_{\mathrm{S}^{2 n}}$ is just the vector bundle

$$
\operatorname{Spin}(2 n+1) \times_{\operatorname{Spin}(2 n)} \mathbf{s}^{2 \mu} \rightarrow \mathrm{S}^{2 n}
$$

with the natural $\operatorname{Spin}(2 n+1)$-invariant connection. Note that, as a hermitian bundle with a hermitian connection, $\mathcal{S}^{2 \mu}$ is the pullback of $\left.\mathcal{S}^{2 \mu}\right|_{\mathrm{S}^{2 n}}$ under the natural projection $\mathbb{R}_{*}^{2 n+1} \rightarrow \mathrm{S}^{2 n}$. Let $L^{2}\left(\mathcal{S}^{2 \mu}\right), L^{2}\left(\left.\mathcal{S}^{2 \mu}\right|_{\mathrm{S}^{2 n}}\right)$ be the $L^{2}$-sections of $\mathcal{S}^{2 \mu}$ and $\left.\mathcal{S}^{2 \mu}\right|_{S^{2 n}}$ respectively. It is clear that $\operatorname{Spin}(2 n+1)$ acts on both $L^{2}\left(\mathcal{S}^{2 \mu}\right)$ and $L^{2}\left(\left.\mathcal{S}^{2 \mu}\right|_{S^{2 n}}\right)$ unitarily. In fact, as a representation of $\operatorname{Spin}(2 n+1), L^{2}\left(\left.\mathcal{S}^{2 \mu}\right|_{S^{2 n}}\right)$ is the induced representation of $\mathbf{s}^{2 \mu}$ from $\operatorname{Spin}(2 n)$ to $\operatorname{Spin}(2 n+1)$; therefore, by the Frobenius reciprocity plus a branching $\operatorname{rule}^{7}$ for $(\operatorname{Spin}(2 n+1)$, $\operatorname{Spin}(2 n))$, one has

$$
L^{2}\left(\left.\mathcal{S}^{2 \mu}\right|_{\mathrm{S}^{2 n}}\right)=\hat{\bigoplus}_{l \geq 0} \mathscr{R}_{l}
$$

where $\mathscr{R}_{l}$ is the irreducible representation of $\operatorname{Spin}(2 n+1)$ with highest weight $(l+|\mu|,|\mu|, \cdots,|\mu|)$. Observe that, if we use $\widetilde{X}$ to denote the horizontal lift of vector field $X$ on $\mathbb{R}_{*}^{2 n+1}$, then the vector field $\left[\widetilde{r \partial_{\alpha}}, \widetilde{r \partial_{\beta}}\right]$ can be shown to be just the right invariant vector field on $\mathbb{R}_{+} \times \operatorname{Spin}(2 n+1)$ whose value at $(r, e)$ (where $e$ is the group identity element) is $\left(0,-i \gamma_{\alpha \beta}\right)$, i.e., $\left(0,-\frac{1}{4}\left[e_{\alpha}, e_{\beta}\right]\right)$. Consequently, the infinitesimal action of $\operatorname{Spin}(2 n+1)$ on $C^{\infty}\left(\mathcal{S}^{2 \mu}\right)$ is just the restriction of $\tilde{\pi}$ to $\operatorname{span}_{\mathbb{R}}\left\{M_{\alpha \beta} \mid 1 \leq \alpha<\beta \leq 2 n+1\right\}=\mathfrak{s o}_{0}(2 n+1)$. It is then clear that $\tilde{\pi}\left(M_{\alpha \beta}\right)$ 's act only on

\footnotetext{
7 See, for example, Theorem 2 of $\S 129$ of Ref. [21].
} 
the angular part of the wave sections - a consequence which can also be deduced from the fact that $\hat{J}_{\alpha \beta}$ 's commute with the multiplication by a smooth function of $r$.

Let $\left\{Y_{l \mathbf{m}}(\Omega)\right\}_{\mathbf{m} \in \mathcal{I}(l)}$ be an orthornormal (say Gelfand-Zeltin) basis for $\mathscr{R}_{l}$, and

$$
l_{\mu}=l+|\mu|+n-1 \text {. }
$$

Then, an orthornormal basis for the energy eigenspace $\mathscr{H}_{I}$ with energy $E_{I}$ is

$$
\left\{\psi_{k l \mathbf{m}}:=R_{k l_{\mu}}(r) Y_{l \mathbf{m}}(\Omega) \mid k+l=I+1, k \geq 1, l \geq 0, \mathbf{m} \in \mathcal{I}(l)\right\}
$$

where $R_{k l_{\mu}} \in L^{2}\left(\mathbb{R}_{+}, r^{2 n} d r\right)$ is a square integrable (with respect to measure $r^{2 n} d r$ ) solution of the radial Schrödinger equation:

$$
\left(-\frac{1}{2 r^{2 n}} \partial_{r} r^{2 n} \partial_{r}+\frac{l_{\mu}\left(l_{\mu}+1\right)-n(n-1)}{2 r^{2}}-\frac{1}{r}\right) R_{k l_{\mu}}=E_{k-1+l} R_{k l_{\mu}} .
$$

Note that $R_{k l_{\mu}}$ is of the form

$$
r^{-n} y_{k l_{\mu}}(r) \exp \left(-\frac{r}{k+l_{\mu}}\right)
$$

with $y_{k l_{\mu}}(r)$ satisfying Eq.

$$
\left(\frac{d^{2}}{d r^{2}}-\frac{2}{k+l_{\mu}} \frac{d}{d r}+\left[\frac{2}{r}-\frac{l_{\mu}\left(l_{\mu}+1\right)}{r^{2}}\right]\right) y_{k l_{\mu}}(r)=0
$$

In term of the generalized Laguerre polynomials,

$$
y_{k l_{\mu}}(r)=c(k, l) r^{l_{\mu}+1} L_{k-1}^{2 l_{\mu}+1}\left(\frac{2}{k+l_{\mu}} r\right)
$$

where $c(k, l)$ is a constant, which can be uniquely determined by requiring $c(k, l)>0$ and $\int_{0}^{\infty}\left|R_{k l_{\mu}}(r)\right|^{2} r^{2 n} d r=1$.

We are now ready to state the following remark.

Remark 1. 1) $\mathscr{H}_{I}$ is the space of square integrable solutions of Eq. $H \psi=E_{I} \psi$.

2) As representation of $\mathfrak{s o}(2 n+1)$,

$$
\mathscr{H}_{I}=\bigoplus_{l=0}^{I} D_{l}
$$

where $D_{l}:=\operatorname{span}\left\{\psi_{(I-l+1) l \mathbf{m}} \mid \mathbf{m} \in \mathcal{I}(l)\right\}$ is the highest weight module with highest weight $(l+|\mu|,|\mu|, \cdots,|\mu|)$.

3) $\left\{\mathscr{H}_{I} \mid I=0,1,2, \ldots\right\}$ is the complete set of (bound) energy eigenspaces.

For the completeness of this review, we state part of Theorem 1 from Ref. [4] below:

Theorem 3. For the $(2 n+1)$-dimensional generalized MICZ-Kepler problem with magnetic charge $\mu$, the following statements are true:

1) The negative energy spectrum is

$$
E_{I}=-\frac{1 / 2}{(I+n+|\mu|)^{2}}
$$


where $I=0,1,2, \ldots$;

2) The Hilbert space $\mathscr{H}(\mu)$ of negative-energy states admits a linear $\operatorname{Spin}(2 n+2)$-action under which there is a decomposition

$$
\mathscr{H}(\mu)=\hat{\bigoplus}_{I=0}^{\infty} \mathscr{H}_{I}
$$

where $\mathscr{H}_{I}$ is the irreducible $\operatorname{Spin}(2 n+2)$-module with highest weight $(I+|\mu|,|\mu|, \cdots,|\mu|, \mu)$;

3) The linear action in part 2) extends the manifest linear action of $\operatorname{Spin}(2 n+1)$, and $\mathscr{H}_{I}$ in part 2) is the energy eigenspace with eigenvalue $E_{I}$ in part 1).

It was shown in Ref. 4] that the bound eigen-states are precisely the ones with negative energy eigenvalues. We would like to remark that, in dimension five, a similar result obtained with a similar method has already appeared in Ref. [18].

\section{B. Twisting}

As we said before, because of the technical intricacy, we need to introduce the notion of twisting. Let us start with the listing of some important spaces used later:

- $\mathscr{H}_{I}$ - the $I$-th bound energy eigenspace;

- $\mathcal{H}$ - the algebraic direct sum of all bound energy eigenspaces;

- $\mathscr{H}$ or $\mathscr{H}(\mu)$ - the completion of $\mathcal{H}$ under the standard $L^{2}$-norm;

- $\mathcal{H}_{l \mathbf{m}}$ - the subspace of $\mathcal{H}$ spanned by $\left\{\psi_{k l \mathbf{m}} \mid k \geq 1, l, \mathbf{m}\right.$ fixed $\}$;

- $\mathscr{H}_{\mathbf{l}}$ - the completion of $\mathcal{H}_{l \mathbf{m}}$ under the standard $L^{2}$-norm.

Note that these spaces are all endowed with the unique hermitian inner product which yields the standard $L^{2}$-norm, i.e.,

$$
\langle\psi, \phi\rangle:=\int_{\mathbb{R}_{*}^{D}}(\psi, \phi) d^{D} x
$$

where $(\psi, \phi)$ is the point-wise hermitian inner product and $d^{D} x$ is the Lebesgue measure.

It is clear from the previous section that

$$
\mathscr{B}:=\left\{\psi_{k l \mathbf{m}} \mid k \geq 1, l \geq 0, \mathbf{m} \in \mathcal{I}(l)\right\}
$$

is an orthonormal basis for both $\mathcal{H}$ and $\mathscr{H}$.

To study the action of $\hat{J}_{A B}$ 's, we need to "twist" $\mathscr{B}, \mathscr{H}_{I}, \mathcal{H}_{l \mathbf{m}}, \mathscr{H}_{\mathbf{l}}, \mathcal{H}$ and $\mathscr{H}$ to get $\tilde{\mathscr{B}}_{1} \tilde{\mathscr{H}}_{I}, \tilde{\mathcal{H}}_{l \mathbf{m}}, \tilde{\mathscr{H}}_{l \mathbf{m}}, \tilde{\mathcal{H}}_{\text {and }}$ $\tilde{\mathscr{H}}$ respectively. It suffices to twist the elements of $\mathscr{B}$. Let $\tau: \mathscr{B} \rightarrow \tilde{\mathscr{B}}$ be defined as follows:

$$
\tau\left(\psi_{k l \mathbf{m}}\right)(r, \Omega):=\left(k+l_{\mu}\right) e^{-i \theta_{k+l_{\mu}} \hat{T}}\left(\frac{1}{\sqrt{r}} \psi_{k l \mathbf{m}}(r, \Omega)\right)
$$




$$
\begin{aligned}
& =\left(k+l_{\mu}\right)^{n+1} \frac{1}{\sqrt{r}} \psi_{k l \mathbf{m}}\left(\left(k+l_{\mu}\right) r, \Omega\right) \\
& \propto r^{l+|\mu|-\frac{1}{2}} L_{k-1}^{2 l_{\mu}+1}(2 r) e^{-r} Y_{l \mathbf{m}}(\Omega)
\end{aligned}
$$

where $\hat{T}=\frac{1}{\sqrt{r}} T \sqrt{r}$, and $\theta_{I}=-\ln I$ for any positive number $I$. For simplicity, we write $\tau\left(\psi_{k l \mathbf{m}}\right)$ as $\tilde{\psi}_{k l \mathbf{m}}$. One can check that

$$
\int_{\mathbb{R}_{*}^{D}}\left(\tilde{\psi}_{k l \mathbf{m}}, \tilde{\psi}_{k l \mathbf{m}}\right) d^{D} x=\int_{\mathbb{R}_{*}^{D}}\left(\psi_{k l \mathbf{m}}, \psi_{k l \mathbf{m}}\right) d^{D} x=1
$$

By using Eq. (40) and the orthogonality identities for the generalized Laguerre polynomials, one can see that $\tilde{\psi}_{k l \mathbf{m}}$ is orthogonal to $\tilde{\psi}_{k^{\prime} l \mathbf{m}}$ when $k \neq k^{\prime}$.

It is now clear how to twist all the relevant spaces listed in the beginning of this subsection. For example,

$$
\tilde{\mathscr{H}}_{I}:=\left\{e^{-i \theta_{I_{\mu}+1} \hat{T}}\left(\frac{1}{\sqrt{r}} \psi\right) \mid \psi \in \mathscr{H}_{I}\right\} .
$$

Since $\mathscr{H}_{I}$ is spanned by $\left\{\psi_{k l \mathbf{m}} \mid k+l=I+1, k \geq 1, l \geq 0, \mathbf{m} \in \mathcal{I}(l)\right\}$, it follows that $\tilde{\mathscr{H}}_{I}$ is spanned by

$$
\left\{\tilde{\psi}_{k l \mathbf{m}} \mid k+l=I+1, k \geq 1, l \geq 0, \mathbf{m} \in \mathcal{I}(l)\right\}
$$

We shall call $\tilde{\mathscr{H}}(\mu)$ the twisted Hilbert space of the bound states for the $(2 n+1)$-dimensional generalized MICZKepler problem with magnetic charge $\mu$. Remark that the twisting map ${ }^{8}$

$$
\tau: \mathscr{H}(\mu) \rightarrow \tilde{\mathscr{H}}(\mu)
$$

is the unique linear isometry which sends $\psi_{k l \mathbf{m}}$ to $\tilde{\psi}_{k l \mathbf{m}}$; moreover, $\tau$ maps all relevant subspaces of $\mathscr{H}(\mu)$ isomorphically onto the corresponding relevant twisted subspaces. Note that $\hat{J}_{\alpha \beta}=\frac{1}{\sqrt{r}} J_{\alpha \beta} \sqrt{r}=J_{\alpha \beta}$ obviously acts on $\tilde{\mathscr{H}}_{I}$ as hermitian operator, so $\mathfrak{r}:=\operatorname{span}\left\{M_{\alpha \beta} \mid 1 \leq \alpha<\beta \leq 2 n+1\right\}=\mathfrak{s o}(2 n+1)$ acts unitarily on $\tilde{\mathscr{H}}_{I}$ via $\tilde{\pi}$.

Recall that for non negative integer $I$, we use $I_{\mu}$ to denote $I+n+|\mu|-1$.

Proposition 1. 1) $\tilde{\psi}_{k l \mathbf{m}}$ is an eigenvector of $\hat{\Gamma}_{-1}$ with eigenvalue $k+l_{\mu}$.

2) $\tilde{\mathscr{H}}_{I}$ is the space of square integrable solutions of Eq. $\hat{\Gamma}_{-1} \psi=\left(I_{\mu}+1\right) \psi$.

3) $\hat{\Gamma}_{-1}$ is a self-adjoint operator on $\tilde{\mathscr{H}}(\mu)$ and $\tilde{\mathscr{H}}_{I}$ is the eigenspace of $\hat{\Gamma}_{-1}$ with eigenvalue $I_{\mu}+1$.

4) As representation of $\mathfrak{r}$,

$$
\tilde{\mathscr{H}}_{I}=\bigoplus_{l=0}^{I} \tilde{D}_{l}
$$

where $\tilde{D}_{l}:=\operatorname{span}\left\{\tilde{\psi}_{(I-l+1) l \mathbf{m}} \mid \mathbf{m} \in \mathcal{I}(l)\right\}$ is the highest weight module with highest weight $(l+|\mu|,|\mu|, \cdots,|\mu|)$.

5) $\tilde{\mathscr{H}}(\mu)=L^{2}\left(\mathcal{S}^{2 \mu}\right)$.

\footnotetext{
${ }^{8}$ It has a basis-free description: for $\psi \in \mathscr{H}_{I}, \tau(\psi)(r, \Omega)=\left(I_{\mu}+1\right)^{n+1} \frac{1}{\sqrt{r}} \psi\left(\left(I_{\mu}+1\right) r, \Omega\right)$.
} 
Proof. 1) The proof is based on the ideas from Ref. [5]. Since

$$
H \psi_{k l \mathbf{m}}=E_{k+l-1} \psi_{k l \mathbf{m}}
$$

we have $\sqrt{r}\left(H-E_{k+l-1}\right) \psi_{k l \mathbf{m}}=0$ which can be rewritten as

$$
\left(\frac{1}{2} \hat{X}-1-E_{k+l-1} \hat{Y}\right)\left(\frac{1}{\sqrt{r}} \psi_{k l \mathbf{m}}\right)=0
$$

where $X$ and $Y$ are given by Eq. (9). In terms of $\hat{\Gamma}_{-1}$ and $\hat{\Gamma}_{D+1}$, we can recast the above equation as

$$
\left(\left(\frac{1}{2}-E_{k+l-1}\right) \hat{\Gamma}_{-1}+\left(\frac{1}{2}+E_{k+l-1}\right) \hat{\Gamma}_{D+1}-1\right)\left(\frac{1}{\sqrt{r}} \psi_{k l \mathbf{m}}\right)=0
$$

Plugging $\psi_{k l \mathbf{m}}=\frac{1}{k+l_{\mu}} \sqrt{r} e^{i \theta_{k+l_{\mu}} \hat{T}}\left(\tilde{\psi}_{k l \mathbf{m}}\right)$ into the above equation and using identities

$$
\left\{\begin{aligned}
e^{-i \theta \hat{T}} \hat{\Gamma}_{-1} e^{i \theta \hat{T}} & =\cosh \theta \hat{\Gamma}_{-1}+\sinh \theta \hat{\Gamma}_{D+1} \\
e^{-i \theta \hat{T}} \hat{\Gamma}_{D+1} e^{i \theta \hat{T}} & =\sinh \theta \hat{\Gamma}_{-1}+\cosh \theta \hat{\Gamma}_{D+1},
\end{aligned}\right.
$$

we arrive at the following equation:

$$
\hat{\Gamma}_{-1} \tilde{\psi}_{k l \mathbf{m}}=\left(k+l_{\mu}\right) \tilde{\psi}_{k l \mathbf{m}} \text {. }
$$

2) Note that the Barut-Bornzin process going from Eq. (44) to Eq. (46) is completely reversible. Therefore, part 2 ) is just a consequence of part 1) of Remark 1.

3) Note that $\hat{\Gamma}_{-1}$ is defined on the dense linear subspace $\tilde{\mathcal{H}}$ of $\tilde{\mathscr{H}}(\mu)$. It is easy to check that

$$
\left\langle\tilde{\psi}_{k^{\prime} l^{\prime} \mathbf{m}^{\prime}}, \hat{\Gamma}_{-1} \tilde{\psi}_{k l \mathbf{m}}\right\rangle=\left\langle\hat{\Gamma}_{-1} \tilde{\psi}_{k^{\prime} l^{\prime} \mathbf{m}^{\prime}}, \tilde{\psi}_{k l \mathbf{m}}\right\rangle
$$

for any $\tilde{\psi}_{k l \mathbf{m}}$ and $\tilde{\psi}_{k^{\prime} l^{\prime} \mathbf{m}^{\prime}}$. Therefore, $\hat{\Gamma}_{-1}$ (To be precise, it should be its closure) is a self-adjoint operator on $\tilde{\mathscr{H}}(\mu)$. In view of part 2), $\tilde{\mathscr{H}}_{I}$ is the eigenspace of $\hat{\Gamma}_{-1}$ with eigenvalue $I_{\mu}+1$.

4) This part is clear due to part 2) of Remark 1 .

$5)$ Recall that $\tilde{\psi}_{k l \mathbf{m}}(r, \Omega)=\tilde{R}_{k l_{\mu}}(r) Y_{l \mathbf{m}}(\Omega)$ where $\tilde{R}_{k l_{\mu}}(r) \propto r^{l+|\mu|-\frac{1}{2}} L_{k-1}^{2 l_{\mu}+1}(2 r) e^{-r}$. By the well-known property for the generalized Laguerre polynomials, for any $l \geq 0,\left\{\tilde{R}_{k l_{\mu}}\right\}_{k=1}^{\infty}$ form an orthonormal basis for $L^{2}\left(\mathbb{R}_{+}, r^{2 n} d r\right)$.

By virtue of Theorem II. 10 of Ref. [19] and Eq. [33),

$$
\begin{aligned}
L^{2}\left(\mathcal{S}^{2 \mu}\right) & =L^{2}\left(\mathbb{R}_{+}, r^{2 n} d r\right) \otimes L^{2}\left(\left.\mathcal{S}^{2 \mu}\right|_{\mathrm{S}^{2 n}}\right) \\
& =\bigoplus_{l=0}^{\infty}\left(L^{2}\left(\mathbb{R}_{+}, r^{2 n} d r\right) \otimes \mathscr{R}_{l}\right)
\end{aligned}
$$

Therefore, $\tilde{\mathscr{B}}$ is an orthonormal basis for $L^{2}\left(\mathcal{S}^{2 \mu}\right)$, consequently $\tilde{\mathscr{H}}(\mu)=L^{2}\left(\mathcal{S}^{2 \mu}\right)$.

We end this subsection with

Remark 2. $\tilde{\mathscr{H}}_{I}$ is the eigenspace of $\tilde{\pi}\left(H_{0}\right)$ with eigenvalue $-\left(I_{\mu}+1\right)$. Here $\tilde{\pi}\left(H_{0}\right)=-\hat{\Gamma}_{-1}$ is viewed as an endomorphism of $\tilde{\mathcal{H}}$. 


\section{REPRESENTATION THEORETICAL ASPECTS - THE FINAL PART}

We start with some notations:

- $G=\operatorname{Spin}(2,2 n+2)$ - the double cover of $\mathrm{SO}_{0}(2,2 n+2)$ characterized by the homomorphism $\pi_{1}\left(\mathrm{SO}_{0}(2,2 n+2)\right)=$ $\mathbb{Z} \oplus \mathbb{Z}_{2} \rightarrow \mathbb{Z}_{2}$ sending $(a, b)$ to $\bar{a}+b ;$

- $\mathfrak{g}_{0}$ - the Lie algebra of $\operatorname{Spin}(2,2 n+2)$;

- $\mathfrak{g}$ - the complexfication of $\mathfrak{g}_{0}$, so $\mathfrak{g}=\mathfrak{s o}(2 n+4)$;

- $H_{0}$ - defined to be $M_{-1,0}$;

- $H_{j}$ - defined to be $-M_{2 j-1,2 j}$ for $1 \leq j \leq n+1$;

- $K:=\operatorname{Spin}(2) \times_{\mathbb{Z}_{2}} \operatorname{Spin}(2 n+2)$ - a maximal compact subgroup of $\operatorname{Spin}(2,2 n+2)$;

- $\mathfrak{k}_{0}$ — the Lie algebra of $K$;

- $\mathfrak{k}$ - the complexfication of $\mathfrak{k}_{0}$, so $\mathfrak{k}=\mathfrak{s o}(2) \oplus \mathfrak{s o}(2 n+2)$;

- $\mathfrak{r}$ - the subalgebra of $\mathfrak{g}$ generated by $\left\{M_{A B} \mid 1 \leq A<B \leq 2 n+1\right\}$, so $\mathfrak{r}_{0}:=\mathfrak{g}_{0} \cap \mathfrak{r}=\mathfrak{s o}_{0}(2 n+1)$;

- $\mathfrak{s}$ - the subalgebra of $\mathfrak{g}$ generated by $\left\{M_{A B} \mid 1 \leq A<B \leq 2 n+2\right\}$, so $\mathfrak{s}_{0}:=\mathfrak{g}_{0} \cap \mathfrak{s}_{=}=\mathfrak{s o}_{0}(2 n+2)$;

- $\mathfrak{s l}(2)$ — the subalgebra of $\mathfrak{g}$ generated by $M_{-1, D+1}, M_{0, D+1}$ and $M_{-1,0}$, so $\mathfrak{s l}_{0}(2):=\mathfrak{g}_{0} \cap \mathfrak{s l}_{(}(2)=\mathfrak{s o}_{0}(2,1)$;

- $U(\mathfrak{s l}(2))$ — the universal enveloping algebra of $\mathfrak{s l}(2)$.

\section{A. $\tilde{\mathcal{H}}$ is a unitary highest weight Harish-Chandra module}

The goal of this subsection is to show that $(\tilde{\pi}, \tilde{\mathcal{H}})$ is a unitary highest weight $(\mathfrak{g}, K)$-module.

Proposition 2. 1) Each $\tilde{\pi}\left(M_{A B}\right)$ maps $\tilde{\mathcal{H}}$ into $\tilde{\mathcal{H}}$, so $(\tilde{\pi}, \tilde{\mathcal{H}})$ is a representation of $\mathfrak{g}$.

2) Each $\tilde{\pi}\left(M_{A B}\right)$ is a hermitian operator on $\tilde{\mathcal{H}}$, so $(\tilde{\pi}, \tilde{\mathcal{H}})$ is a unitary representation of $\mathfrak{g}$.

3) $\left(\left.\tilde{\pi}\right|_{\mathfrak{s l}(2)}, \tilde{\mathcal{H}}_{l \mathbf{m}}\right)$ is the discrete series representation of $\mathfrak{s o}(2,1)$ with highest weight $-l_{\mu}-1$.

Proof. 1) We follow the convention of Ref. [20] for describing the root space of $\mathfrak{g}=\mathfrak{s o}(2 n+4)$. Take as a basis of the Cartan sub-algebra of $\mathfrak{g}$ the following elements:

$$
H_{0}=M_{-1,0}, \quad H_{j}=-M_{2 j-1,2 j}, \quad j=1, \cdots, n+1
$$

Let $\eta, \eta^{\prime}= \pm 1$. We take the following root vectors:

$$
E_{\eta e^{j}+\eta^{\prime} e^{k}}=\frac{1}{2}\left(M_{2 j-1,2 k-1}+i \eta M_{2 j, 2 k-1}+i \eta^{\prime} M_{2 j-1,2 k}-\eta \eta^{\prime} M_{2 j, 2 k}\right)
$$


where $0 \leq j<k \leq n+1$. This way we obtain a Cartan basis for $\mathfrak{g}$. Therefore, for $\psi_{I} \in \tilde{\mathscr{H}}_{I}$, we have

$$
\begin{aligned}
\tilde{\pi}\left(H_{0}\right)\left(\tilde{\pi}\left(E_{\alpha}\right)\left(\psi_{I}\right)\right) & =\left(-I_{\mu}-1+\alpha_{0}\right) \tilde{\pi}\left(E_{\alpha}\right)\left(\psi_{I}\right) \\
& =\left(-\left(I-\alpha_{0}\right)_{\mu}-1\right) \tilde{\pi}\left(E_{\alpha}\right)\left(\psi_{I}\right)
\end{aligned}
$$

where $\alpha_{0}$ (which can be 0 , or -1 or 1 ) is the 0 -th component of $\alpha$. It is not hard to see that $\tilde{\pi}\left(E_{\alpha}\right)\left(\psi_{I}\right)$ is square integrable ${ }^{9}$, so in view of part 2) of Proposition 11. Eq. (47) implies that $\tilde{\pi}\left(E_{\alpha}\right)\left(\psi_{I}\right) \in \tilde{\mathscr{H}}_{I-\alpha_{0}}$. (Here $\mathscr{H}_{-1}=0$.) Therefore, $\tilde{\pi}\left(E_{\alpha}\right)$ maps any $\tilde{\mathscr{H}}_{I}$, hence $\tilde{\mathcal{H}}$, into $\tilde{\mathcal{H}}$. By a similar argument, one can show that $\tilde{\pi}\left(H_{i}\right)$ maps $\tilde{\mathcal{H}}$ into itself. Since $H$ 's and $E$ 's form a basis for $\mathfrak{g}$, this implies that $\tilde{\pi}\left(M_{A B}\right)$ maps $\tilde{\mathcal{H}}$ into itself.

2) It is equivalent to checking that each $\hat{J}_{A B}:=\frac{1}{\sqrt{r}} J_{A B} \sqrt{r}$ is an hermitian operator on $\tilde{\mathcal{H}}$. First of all, it is not hard to see that, when $\mathcal{O}=\pi_{\alpha}, r, \frac{1}{r}, \sqrt{r}, \frac{1}{\sqrt{r}}$, we always have

$$
\left\langle\psi_{1}, \mathcal{O} \psi_{2}\right\rangle=\left\langle\mathcal{O} \psi_{1}, \psi_{2}\right\rangle
$$

for any $\psi_{1}, \psi_{2}$ in $\tilde{\mathcal{H}}$. It is equally easy to see that Eq. (48) is always true for any $\psi_{1}, \psi_{2}$ in $\tilde{\mathcal{H}}$ when $\mathcal{O}$ is $\hat{\Gamma}_{\alpha}=\sqrt{r} \pi_{\alpha} \sqrt{r}$, $\hat{X}=\sqrt{r} \pi^{2} \sqrt{r}+\frac{c}{r}$, or $\hat{Y}=r$. It is then clear from definitions (9) and (10) that Eq. (48) is always true for any $\psi_{1}, \psi_{2}$ in $\tilde{\mathcal{H}}$ when $\mathcal{O}=\hat{J}_{A B}$.

$3)$ Let us first show that $\tilde{\pi}\left(M_{-1, D+1}\right), \tilde{\pi}\left(M_{0, D+1}\right)$ and $\tilde{\pi}\left(M_{-1,0}\right)$ map each $\tilde{\psi}_{k l \mathbf{m}}$ into $\tilde{\mathcal{H}}_{l \mathbf{m}}$, so they indeed map $\tilde{\mathcal{H}}_{l \mathbf{m}}$ into $\tilde{\mathcal{H}}_{l \mathbf{m}}$. This is obvious for $\tilde{\pi}\left(M_{-1,0}\right)$ because $\tilde{\pi}\left(M_{-1,0}\right)\left(\tilde{\psi}_{k l \mathbf{m}}\right)=-\hat{\Gamma}_{-1} \tilde{\psi}_{k l \mathbf{m}}=-\left(k+l_{\mu}\right) \tilde{\psi}_{k l \mathbf{m}}$. Next, we introduce

$$
E_{ \pm}=\frac{1}{\sqrt{2}}\left(M_{-1, D+1} \pm i M_{0, D+1}\right),
$$

then one can check from Eq. (30) that $\left[M_{-1,0}, E_{ \pm}\right]= \pm E_{ \pm}$. Therefore

$$
\tilde{\pi}\left(M_{-1,0}\right)\left(\tilde{\pi}\left(E_{ \pm}\right)\left(\tilde{\psi}_{k l \mathbf{m}}\right)\right)=\left(-k-l_{\mu} \pm 1\right) \tilde{\pi}\left(E_{ \pm}\right)\left(\tilde{\psi}_{k l \mathbf{m}}\right)
$$

where $\tilde{\pi}\left(E_{ \pm}\right)=\frac{1}{\sqrt{2}}\left(\hat{T} \pm i \hat{\Gamma}_{D+1}\right)$. It is not hard to see that $\tilde{\pi}\left(E_{ \pm}\right)\left(\tilde{\psi}_{k l \mathbf{m}}\right)$ is square integrable. In view of part 2$)$ of Proposition [1, we conclude that $\tilde{\pi}\left(E_{ \pm}\right)\left(\tilde{\psi}_{k l \mathbf{m}}\right)$ must be proportional to $\tilde{\psi}_{(k \mp 1) l \mathbf{m}} \cdot\left(\right.$ Here, by convention, $\tilde{\psi}_{0 l \mathbf{m}}=0$.) Therefore, operators $\tilde{\pi}\left(E_{ \pm}\right)$map $\tilde{\psi}_{k l \mathbf{m}}$ into $\tilde{\mathcal{H}}_{l \mathbf{m}}$. This proves that $\left(\left.\tilde{\pi}\right|_{\mathfrak{s l}(2)}, \tilde{\mathcal{H}}_{l \mathbf{m}}\right)$ is a representation of $\mathfrak{s l}(2)$.

In view of the fact that $\tilde{\pi}\left(M_{-1,0}\right)\left(\tilde{\psi}_{1 l \mathbf{m}}\right)=-\left(l_{\mu}+1\right) \tilde{\psi}_{1 l \mathbf{m}} \neq 0$, we conclude that $U(\mathfrak{s l}(2)) \cdot \tilde{\psi}_{1 l \mathbf{m}}$ is a nontrivial unitary highest weight representation of the non-compact real Lie algebra $\mathfrak{s l}_{0}(2)$, hence must be the discrete series representation with highest weight $-\left(l_{\mu}+1\right)$. Since $U(\mathfrak{s l}(2)) \cdot \tilde{\psi}_{1 l \mathbf{m}} \subset \tilde{\mathcal{H}}_{l \mathbf{m}}$, and $\operatorname{dim}\left(\mathscr{H}_{I} \cap U(\mathfrak{s l}(2)) \cdot \tilde{\psi}_{1 l \mathbf{m}}\right)=$ $\operatorname{dim}\left(\mathscr{H}_{I} \cap \tilde{\mathcal{H}}_{l \mathbf{m}}\right)$ for all $I \geq 0$, we conclude that $U(\mathfrak{s l}(2)) \cdot \tilde{\psi}_{1 l \mathbf{m}}=\tilde{\mathcal{H}}_{l \mathbf{m}}$. Therefore, $\tilde{\mathcal{H}}_{l \mathbf{m}}$ is a unitary highest weight $\mathfrak{s l}(2)$-module with highest weight $-l_{\mu}-1$, which in fact is a unitary highest weight $(\mathfrak{s l}(2), \operatorname{Spin}(2))$-module. Then $\tilde{\mathscr{H}}_{l \mathbf{m}}$ must be the discrete series representation of $\operatorname{Spin}(2,1)$ with highest weight $-l_{\mu}-1$.

\footnotetext{
9 The convergence of the integral near infinity is clear because of the exponential decay as $r \rightarrow \infty$. The convergence of the integral near the origin of $\mathbb{R}_{*}^{D}$ in the general case is clear from the counting of powers of $r$, and the remaining case when $n=1, \mu=0$ and $\psi_{I}=\tilde{\psi}_{k 00}$ needs a separate but equally easy argument.
} 
To continue the discussion on representations, we prove the following proposition.

Proposition 3. 1) $\left(\left.\tilde{\pi}\right|_{\mathfrak{s}}, \tilde{\mathscr{H}}_{I}\right)$ is an irreducible unitary representation of $\mathfrak{s}$, in fact, it is the highest weight representation with highest weight $(I+|\mu|,|\mu|, \ldots,|\mu|, \mu)$.

2) The unitary action of $\mathfrak{k}_{0}$ on $\tilde{\mathcal{H}}$ can be lifted to a unique unitary action of $K$ under which

$$
\tilde{\mathcal{H}}=\bigoplus_{l=0}^{\infty}\left(D\left(-l_{\mu}-1\right) \otimes D^{l}\right)
$$

where $D^{l}$ is the irreducible module of $\operatorname{Spin}(2 n+2)$ with highest weight $(l+|\mu|,|\mu|, \cdots,|\mu|, \mu)$ and $D\left(-l_{\mu}-1\right)$ is the irreducible module of $\operatorname{Spin}(2)$ with weight $-l_{\mu}-1$.

3) $\tilde{\mathcal{H}}$ is a unitary $(\mathfrak{g}, K)$-module.

4) $(\tilde{\pi}, \tilde{\mathcal{H}})$ is irreducible; in fact, it is the unitary highest weight module of $\mathfrak{g}$ with highest weight

$$
(-(n+|\mu|),|\mu|, \cdots,|\mu|, \mu)
$$

Proof. 1) Recall that $\mathfrak{s}$ is the $\mathfrak{s o}(2 n+2)$ Lie sub algebra of $\mathfrak{g}$ generated by

$$
\left\{H_{i}, E_{ \pm e^{j} \pm e^{k}} \mid 1 \leq i \leq n+1,1 \leq j<k \leq n+1\right\}
$$

and $\mathfrak{s}_{0}:=\mathfrak{s} \cap \mathfrak{g}_{0}$ is the compact real form of $\mathfrak{s}$. Since $H_{0}$ commutes with any element in $\mathfrak{s}$, in view of Remark 2 , we conclude that each $\tilde{\mathscr{H}}_{I}$ is invariant under $\tilde{\pi}(\mathfrak{s})$, i.e., $\left(\left.\tilde{\pi}\right|_{\mathfrak{s}}, \tilde{\mathscr{H}}_{I}\right)$ is a representation of $\mathfrak{s}$.

Inside $\mathfrak{s}$ there is an $\mathfrak{s o}(2 n+1)$ Lie sub algebra $\mathfrak{r}$. Note that $H_{1}, \ldots, H_{n}$ are the generators of a Cartan subalgebra of $\mathfrak{r}$, and $H_{1}, \ldots, H_{n+1}$ are the generators of a Cartan subalgebra of $\mathfrak{s}$. Recall from part 4) of Proposition 1

$$
\left(\left.\tilde{\pi}\right|_{\mathfrak{r}}, \tilde{\mathscr{H}}_{I}\right)=\bigoplus_{l=0}^{I} \tilde{D}_{l}
$$

where $\tilde{D}_{l}$ is the highest weight $\mathfrak{r}$-module with highest weight $(l+|\mu|,|\mu|, \cdots,|\mu|)$.

By applying the branching rule ${ }^{10}$ for $(\mathfrak{s}, \mathfrak{r})$, one finds that there are only two solutions to Eq. $(\underline{501}):\left(\left.\tilde{\pi}\right|_{\mathfrak{s}}, \tilde{\mathscr{H}}_{I}\right)$ is the highest weight module of $\mathfrak{s}$ with highest weight equal to either $(I+|\mu|,|\mu|, \cdots,|\mu|, \mu)$ or $(I+|\mu|,|\mu|, \cdots,|\mu|,-\mu)$. Let $\tilde{\psi}_{1 I \mathbf{I}} \in \tilde{\mathscr{H}}_{I}$ be an $\mathfrak{s - h i g h e s t ~ w e i g h t ~ v e c t o r , ~ w h i c h ~ i s ~ a s s u m e d ~ t o ~ h a v e ~ u n i t ~ n o r m . ~ S i n c e ~} \tilde{\pi}\left(H_{n+1}\right)=\hat{A}_{D}$, we have either $\hat{A}_{D} \tilde{\psi}_{1 I \mathbf{I}}=\mu \tilde{\psi}_{1 I \mathbf{I}}$ or $\hat{A}_{D} \tilde{\psi}_{1 I \mathbf{I}}=-\mu \tilde{\psi}_{1 I \mathbf{I}}$. To determine the sign, we only need to show that $\left\langle\tilde{\psi}_{1 I \mathbf{I}}, A_{D} \tilde{\psi}_{1 I \mathbf{I}}\right\rangle=\mu$. Note that $A_{D}=i\left[\Gamma_{D}, \Gamma_{D+1}\right]=i\left[\Gamma_{D}, \Gamma_{-1}-r\right]=i\left[\Gamma_{D}, \Gamma_{-1}\right]-x_{D}$ and $\tilde{\psi}_{1 I \mathbf{I}}$ is an eigenvector of $\hat{\Gamma}_{-1}$, so

$$
\begin{aligned}
\left\langle\tilde{\psi}_{1 I \mathbf{I}}, \hat{A}_{D} \tilde{\psi}_{1 I \mathbf{I}}\right\rangle & =-\left\langle\tilde{\psi}_{1 I \mathbf{I}}, x_{D} \tilde{\psi}_{1 I \mathbf{I}}\right\rangle \\
& =-\int_{\mathbb{R}_{*}^{D}} x_{D}\left|\tilde{\psi}_{1 I \mathbf{I}}(r, \Omega)\right|^{2} d^{D} x .
\end{aligned}
$$

One can show that ${ }^{11}$, up to a multiplicative constant, $\tilde{\psi}_{1 I \mathbf{I}}(r, \Omega)$ is equal to

$$
r^{I_{\mu}-n+\frac{1}{2}} e^{-r} \cdot(\sin \theta)^{-(n-1)}(1-\cos \theta)^{\frac{I_{\mu}+\mu}{2}}(1+\cos \theta)^{\frac{I_{\mu}-\mu}{2}} \cdot Z\left(\theta_{1}, \ldots, \theta_{D-3}, \phi\right) .
$$

10 See, for example, Theorem 3 of $\$ 129$ of Ref. [21].

11 To be more specific, one needs to generalize the work of Ref. [23]. Since we are only interested in a sign, we choose to skip the details here. 
Then

$$
\begin{aligned}
\left\langle\tilde{\psi}_{1 I \mathbf{I}}, \hat{A}_{D} \tilde{\psi}_{1 I \mathbf{I}}\right\rangle & =-\int_{\mathbb{R}_{x}^{D}} x_{D}\left|\tilde{\psi}_{1 I \mathbf{I}}(r, \Omega)\right|^{2} d^{D} x \\
& =-\frac{\int_{0}^{\infty} r^{2 I_{\mu}+2} e^{-2 r} d r}{\int_{0}^{\infty} r^{2 I_{\mu}+1} e^{-2 r} d r} \cdot \frac{\int_{0}^{\pi} \cos \theta(1-\cos \theta)^{I_{\mu}+\mu}(1+\cos \theta)^{I_{\mu}-\mu} \sin \theta d \theta}{\int_{0}^{\pi}(1-\cos \theta)^{I_{\mu}+\mu}(1+\cos \theta)^{I_{\mu}-\mu} \sin \theta d \theta} \\
& =-\frac{\Gamma\left(2 I_{\mu}+3\right)}{2 \cdot \Gamma\left(2 I_{\mu}+2\right)} \cdot \frac{\int_{-1}^{1} x(1-x)^{I_{\mu}+\mu}(1+x)^{I_{\mu}-\mu} d x}{\int_{-1}^{1}(1-x)^{I_{\mu}+\mu}(1+x)^{I_{\mu}-\mu} d x} \\
& =-\left(\int_{-1}^{1}(1-x)^{I_{\mu}+\mu}(1+x)^{I_{\mu}+1-\mu} d x\right. \\
& =-\left(I_{\mu}^{1}(1-x)^{I_{\mu}+\mu}(1+x)^{I_{\mu}-\mu} d x\right. \\
& =-\left(2 \cdot \frac{B\left(I_{\mu}+1+\mu, I_{\mu}+2-\mu\right)}{B\left(I_{\mu}+1+\mu I_{\mu}+1-\mu\right)}-1\right) \\
&
\end{aligned}
$$

Part 1) is done.

2) Since $\tilde{\mathscr{H}}_{l}$ is the space of square integrable solutions of Eq. $\hat{\Gamma}_{-1} \psi=\left(l_{\mu}+1\right) \psi$ and $\tilde{\pi}\left(H_{0}\right)=-\hat{\Gamma}_{-1}$, as a $\mathfrak{k}$-module, $\tilde{\mathscr{H}}_{l}=D\left(-l_{\mu}-1\right) \otimes D^{l}$ where $D^{l}$ is the irreducible module of $\operatorname{Spin}(2 n+2)$ with highest weight $(l+|\mu|,|\mu|, \cdots,|\mu|, \mu)$ and $D\left(-l_{\mu}-1\right)$ is the irreducible module of $\operatorname{Spin}(2)$ with weight $-l_{\mu}-1$. Since $\mu$ is a half integer, the irreducible unitary action of $\mathfrak{k}_{0}$ on $\tilde{\mathscr{H}}_{l}$ can be promoted to a unique irreducible unitary action of $K$. Therefore, $\tilde{\mathcal{H}}$ is a unitary $K$-module and has the following decomposition into isotypic components of $K$ :

$$
\tilde{\mathcal{H}}=\bigoplus_{l=0}^{\infty} \tilde{\mathscr{H}}_{l}=\bigoplus_{l=0}^{\infty}\left(D\left(-l_{\mu}-1\right) \otimes D^{l}\right)
$$

3) From the definition, it is clear that the action of $K$ on $\tilde{\mathcal{H}}$ is compatible with that of $\mathfrak{g}$ on $\tilde{\mathcal{H}}$, and its linearization agrees with the action of $\mathfrak{k}_{0}$. Part 2) says that $\tilde{\mathcal{H}}$ is $K$-finite. Therefore, $\tilde{\mathcal{H}}$ is a unitary $(\mathfrak{g}, K)$-module.

4) Let $v \neq 0$ be a vector in $\tilde{\mathscr{H}}_{0}$ with $\mathfrak{g}$-weight $(-(n+|\mu|),|\mu|, \ldots,|\mu|, \mu)$. Since this weight is the highest among all weights with a nontrivial weight vector in $\tilde{\mathcal{H}}, V:=U(\mathfrak{g}) \cdot v \subset \tilde{\mathcal{H}}$ is the unitary highest weight $\mathfrak{g}$-module with highest weight $(-(n+|\mu|),|\mu|, \ldots,|\mu|, \mu)$. Since $\tilde{\mathscr{H}}_{l}$ is irreducible under $\mathfrak{s} \subset \mathfrak{g}$, either $\tilde{\mathscr{H}}_{l} \subset V$ or $\tilde{\mathscr{C}}_{l} \cap V=0$, so in particular $\tilde{\mathscr{H}}_{0} \subset V$. We claim that $\tilde{\mathscr{H}}_{l} \subset V$ for any $l \geq 0$, consequently $V=\tilde{\mathcal{H}}$ and then part 4$)$ is done. To prove the claim, we note that $U(\mathfrak{s l}(2)) \cdot v$ must be the discrete series representation of $\mathfrak{s l}(2)$ with highest $\left(H_{0^{-}}\right)$weight $-(n+|\mu|)$ because it is a nontrivial unitary highest weight representation of the non-compact Lie algebra $\mathfrak{s l}_{0}(2)$. In view of the fact that $\tilde{\mathscr{H}}_{l}$ is the eigenspace of $\tilde{\pi}\left(H_{0}\right)$ with eigenvalue $-\left(l_{\mu}+1\right), \tilde{\mathscr{H}} \tilde{\mathscr{l}}_{l} \cap(U(\mathfrak{s l}(2)) \cdot v)=\operatorname{span}\left\{\tilde{\pi}\left(E_{-}^{l}\right)(v)\right\}$ must be one-dimensional. Then $\tilde{\mathscr{H}}_{l} \cap V \neq 0$ because $\operatorname{dim}\left(\tilde{\mathscr{H}}_{l} \cap V\right) \geq \operatorname{dim}\left(\tilde{\mathscr{H}}_{l} \cap(U(\mathfrak{s l}(2)) \cdot v)\right)=1$. 


\section{B. Proof of Theorem 1}

Viewing the twisting map $\tau$ as an equivalence of representations, we get a representation $\pi$ of $\mathfrak{g}$ equivalent to $\tilde{\pi}$. Then the two propositions proved in the previous subsection are true if we drop all "tilde" there. Thus $\mathcal{H}$ is the unitary highest weight $\left(\mathfrak{g}_{0}, K\right)$-module with highest weight $(-(n+|\mu|),|\mu|, \ldots,|\mu|, \mu)$. By a standard theorem of HarishChandra $^{12}$, we know that $\mathscr{H}$ is the unitary highest weight $G$-module with highest weight $(-(n+|\mu|),|\mu|, \ldots,|\mu|, \mu)$ such that $(\pi, \mathcal{H})$ is the underlying $\left(\mathfrak{g}_{0}, K\right)$-module. One can check that this highest weight module occurs at the first reduction point of the Enright-Howe-Wallach classification diagram ${ }^{13}$. So part 1) is done. Part 2) of Theorem 1 is just a consequence of part 3) of Proposition 2, and part 3) of Theorem 1 is just a consequence of part 2) of Proposition 3 .

\section{APPENDIX A: GEOMETRICALLY TRANSPARENT DESCRIPTION}

The purpose of this appendix is to give a geometrically transparent description of the unitary highest weight module of $\operatorname{Spin}(2,2 n+2)$ with highest weight $(-(n+|\mu|),|\mu|, \cdots,|\mu|, \mu)$.

As usual, we assume $n \geq 1$ is an integer and let $\mathcal{S}^{2 \mu}$ be the pullback bundle under the natural retraction $\mathbb{R}_{*}^{2 n+1} \rightarrow \mathrm{S}^{2 n}$ of the vector bundle $\operatorname{Spin}(2 n+1) \times \operatorname{Spin}(2 n) \mathbf{s}^{2 \mu} \rightarrow \mathrm{S}^{2 n}$ with the natural $\operatorname{Spin}(2 n+1)$-invariant connection. Let $d^{D} x$ be the Lebesgue measure on $\mathbb{R}^{2 n+1}$. As is standard in geometry, we use $L^{2}\left(\mathcal{S}^{2 \mu}\right)$ to denote the Hilbert space of square integrable (with respect to $\left.d^{D} x\right)$ sections of $\mathcal{S}^{2 \mu}$. We have shown that $\tilde{\mathscr{H}}(\mu)=L^{2}\left(\mathcal{S}^{2 \mu}\right)$, therefore, $\left(\tilde{\pi}, L^{2}\left(\mathcal{S}^{2 \mu}\right)\right)$ is the unitary highest weight module of $\operatorname{Spin}(2,2 n+2)$ with highest weight $(-(n+|\mu|),|\mu|, \cdots,|\mu|, \mu)$. To describe the infinitesimal action of $\operatorname{Spin}(2,2 n+2)$ on $C^{\infty}\left(\mathcal{S}^{2 \mu}\right)$, it suffices to describe how $M_{\alpha, 0}, M_{D+1,0}$ and $M_{-1,0}$ act as differential operators. It is easy to see that $M_{\alpha, 0}, M_{D+1,0}$ and $M_{-1,0}$ are equal to $i \sqrt{r} \nabla_{\alpha} \sqrt{r}, \frac{1}{2}\left(\sqrt{r} \Delta_{\mu} \sqrt{r}+r-\frac{c}{r}\right)$ and $\frac{1}{2}\left(\sqrt{r} \Delta_{\mu} \sqrt{r}-r-\frac{c}{r}\right)$ respectively. Here $\Delta_{\mu}$ is the Laplace operator twisted by $\mathcal{S}^{2 \mu}$. For example, for $\psi \in C^{\infty}\left(\mathcal{S}^{2 \mu}\right)$, we have

$$
\left(M_{\alpha, 0} \cdot \psi\right)(r, \Omega)=i \sqrt{r} \nabla_{\alpha}(\sqrt{r} \psi(r, \Omega))
$$

[1] H. McIntosh and A. Cisneros, Degeneracy in the presence of a magnetic monopole, J. Math. Phys. 11 (1970), 896-916.

[2] D. Zwanziger, Exactly soluble nonrelativistic model of particles with both electric and magnetic charges, Phys. Rev. 176 (1968), 1480-1488.

[3] T. Iwai, The geometry of the SU(2) Kepler problem, J. Geom. Phys. 7 (1990), 507-535.

\footnotetext{
12 See, for example, Theorem 7 on page 71 of Ref. [22]

13 Page 101, Ref. [10]. In our case $z=A\left(\lambda_{0}\right)=n+1$. It is in Case II when $\mu=0$. For $\mu \neq 0$, it is in Case I for $p=n+1$ or in Case III depending on the sign of $\mu$. See pages $125-126$, Ref. [10]. Note that, while there are two reduction points when $\mu=0$, there is only one reduction point when $\mu \neq 0$.
} 
[4] G. W. Meng, MICZ-Kepler problems in all dimensions. J. Math. Phys. 48 (2007), 032105. E-print, arXiv:math-ph/0507028.

[5] A. Barut and G. Bornzin, SO(4,2)-Formulation of the Symmetry Breaking in Relativistic Kepler Problems with or without Magnetic Charges, J. Math. Phys. 12 (1971), 841-843.

[6] M V Pletyukhov and E A Tolkachev, SO(6,2) dynamical symmetry of the SU(2) MIC-Kepler problem, J. Phys. A: Math. Gen. 32 (1999), 249-253.

[7] G. W. Meng, Generalized MICZ-Kepler Problems and Unitary Highest Weight Modules - II. E-print, arXiv:0704.2936.

[8] H. P. Jakobsen, The last possible place of unitarity for certain highest weight modules, Math. Ann. 256 (1981), no. 4, 439-447.

[9] H. P. Jakobsen, Hermitian symmetric spaces and their unitary highest weight modules, J. Funct. Anal. 52 (1983), no. 3, 385-412.

[10] T. Enright, R. Howe and N. Wallach, A classification of unitary highest weight modules, Representation theory of reductive groups, Progress in Math. 40, Birkhäuser (1983), 97-143.

[11] G. W. Meng, in preparation.

[12] R. Howe and E. Tan, Homogeneous functions on light cones, Bull. Amer. Math. Soc. 28 (1993), 1-74.

[13] T. Kobayashi and B. Ørsted, Analysis on the minimal representation of $\mathrm{O}(p, q)$. I. Realization via conformal geometry, Adv. Math. 180 (2003), no. 2, 486-512.

[14] T. Enright and N. Wallach, Embeddings of unitary highest weight representations and generalized Dirac operators, Math. Ann. 307 (1997), 627-646.

[15] L. Feher, Dynamical O(4) symmetry in the asymptotic field of the Prasad-Sommerfield monopole, J. Phys. A: Math. Gen. 19 (1986), 1259-1270.

[16] G. W. Meng, Dirac and Yang monopoles revisited. E-print, arXiv:math-ph/0409051. To appear in Central European Journal of Physics.

[17] I. I. Cotaescu: Generalized Dirac monopoles in non-Abelian Kaluza-Klein theories. Nucl. Phys. B 719 (2005) $140-164$.

[18] P. Levay, On the SU(2) Kepler problem, Journal of Mathematical Physics 41 (2000), 7382-7385.

[19] M. Reed and B. Simon, Methods of Modern Mathematical Physics, Vol. 1, Academic Press Inc., London (1980).

[20] H. Georgi, Lie Algebras in Particle Physics, Benjamin, London (1982).

[21] D. P. Zelobenko, Compact Lie groups and their representations, Translations of mathematical monographs v. 40, American Mathematical Society, 1973.

[22] Representation Theory and Automorphic Forms, T.N. Bailey, A.W. Knapp, eds, Proceedings of Symposia in Pure Mathematics, Vol. 61, Amer. Math. Soc. 1997.

[23] T. T. Wu and Chen Ning Yang, Dirac monopole without strings: monopole harmonics, Nucl. Phys. B 107(1976), 365-380. 